\title{
Insights into the mechanism of Arnebia euchroma on leukemia via network pharmacology approach
}

\section{Biting Wang}

East China University of Science and Technology

\section{Zengrui Wu}

East China University of Science and Technology

Jiye Wang

East China University of Science and Technology

\section{Weihua Li}

East China University of Science and Technology

Guixia Liu

East China University of Science and Technology

\section{Bo Zhang}

Shihezi University

Yun Tang ( $\nabla$ ytang234@ecust.edu.cn )

East China University of Science and Technology https://orcid.org/0000-0003-2340-1109

\section{Research}

Keywords: Arnebia euchroma; Concentration, Leukemia, Traditional Chinese Medicine, Network pharmacology

Posted Date: April 30th, 2020

DOI: https://doi.org/10.21203/rs.3.rs-24955/v1

License: (c) (1) This work is licensed under a Creative Commons Attribution 4.0 International License. Read Full License

Version of Record: A version of this preprint was published at BMC Complementary Medicine and Therapies on October 27th, 2020. See the published version at https://doi.org/10.1186/s12906-02003106-z. 


\section{Insights into the mechanism of Arnebia euchroma on leukemia via network pharmacology approach}

Biting Wang ${ }^{1}$, Zengrui $\mathrm{Wu}^{1}$, Jiye Wang ${ }^{1}$, Weihua $\mathrm{Li}^{1}$, Guixia Liu ${ }^{1}$, Bo Zhang ${ }^{* 2}$ and Yun Tang ${ }^{* 1}$

*Correspondence:

${ }^{1}$ Shanghai Key

Laboratory of New Drug

Design, School of

Pharmacy, East China

University of Science and

Technology, Shanghai 200237, China. E-mail: ytang234@ecust.edu.cn.

${ }^{2}$ Key Laboratory of Xinjiang Phytomedicine Resource and Utilization, Ministry of Education, School of Pharmacy, Shihezi University, Shihezi 832002, China. Email:

bozhang_lzu@126.com.

\begin{abstract}
Background: Arnebia euchroma (A. euchroma), a traditional Chinese medicine (TCM) for the treatment of blood diseases. In recent years, more and more researches have been conducted on the anti-tumor effect of shikonin and its derivatives, the major active components of A. euchroma. However, the underlying mechanism of action (MoA) for all the ingredients of A. euchroma on leukemia has not been explored systematically.

Methods: In this study, we tried to analyze the MoA of A. euchroma on leukemia via network pharmacology approach. Firstly, the chemical components and their concentrations in A. euchroma as well as leukemia-related targets were collected. Next, we predicted compound-target interactions (CTIs) via our balanced substructure-drug-target network-based inference (bSDTNBI) method. The known and predicted targets of A. euchroma and leukemia-related targets were merged together to construct A. euchroma-leukemia protein-protein interactions (PPIs) network. Then, weighted compound-target bipartite network was constructed according to combination of eight central attributes with concentrations information via Cytoscape. Additionally, molecular docking simulation was performed to determine the binding efficiency of compounds with targets.
\end{abstract}

Results: A total of 65 components of A. euchroma were obtained and 27 of them with concentration information, which involved in 157 targets, 779 compoundtarget interactions (CTIs), including 129 known CTIs (KCTIs) and 650 predicted CTIs (PCTIs). Following the analysis of $A$. euchroma-leukemia PPI network, 37 targets were used to construct the weighted compound-target bipartite network and further KEGG pathway analysis. We found that A. euchroma candidate targets were significantly associated with several apoptosis and inflammation-related biological pathways, such as MAPK signaling pathway, PI3K-Akt signaling pathway, IL-17 signaling pathway, T cell receptor signaling pathway. Moreover, molecular docking simulation demonstrated that there were 8 pairs of PCTIs had the strong binding free energy.

Conclusions: This network pharmacology-based study considered the information of component concentration, in combination with molecular docking, deciphered that the efficacy of A. euchroma in the treatment of leukemia may be mainly attributed to 10 targets and 14 components, which were associated with inhibiting leukemia cell survival and inducing apoptosis, relieving inflammatory environment and inhibiting angiogenesis.

Key Words: Arnebia euchroma; Concentration; Leukemia; Traditional Chinese Medicine; Network pharmacology 


\section{Background}

Arnebia euchroma (Royle) Jonst., also called Xinjiang Zicao, is a traditional Chinese medicine (TCM) for the treatment of blood diseases. The effects of A. euchroma include heat cleaning and blood cooling (Qingre Liangxue in Chinese), to activate blood and detoxification (Huoxue Jiedu in Chinese). Modern studies have demonstrated that $A$. euchroma has multiple pharmacological activities, such as anti-pathogenic, antiinflammatory, anti-allergic, and anti-tumor effects [1-3]. The components of $A$. euchroma consist of two major categories. One is hydrophilic components, mainly a mixture of polysaccharides and glycoproteins; the other is lipophilic substances with a variety of biological activities, including naphthoquinones, alkaloids, monoterpene phenol and benzoquinones, organic acid ester, and so on. Among them, typical naphthoquinones include shikonin and a series of derivatives [4], which have a common scaffold of 5,8-dihydroxy-2-isohexene-1,4-naphthoquinone. According to the optical configuration of the chiral center in the side chain, naphthoquinones can be divided into two subtypes: $R$-configuration (named shikonin) and $S$-configuration (named alkannin) (see Supplementary Figure S1) [5]. Studies have shown that naphthoquinones are the major active ingredients of A. euchroma that exert its pharmacological effects, whose concentration is no more than $7 \%[6,7]$.

Leukemia is a common malignancy in children and adults originating from pluripotent hematopoietic stem cells. Due to various reasons, leukemia cells undergo enhanced selfrenewal, over proliferation, and blocked differentiation and apoptosis. Abnormal 
immature cells proliferate and accumulate in bone marrow and other hematopoietic tissues, leading to the suppression of normal hematopoiesis and infiltration of other organs and tissues. According to the types of cells affected and the developmental stage of the originating cells, leukemia is classified into four major categories: acute myeloid leukemia (AML), acute lymphoblastic leukemia (ALL), chronic myeloid leukemia (CML), and chronic lymphoblastic leukemia (CLL). It is well known that leukemic cells possess some biological properties, such as differentiation arrest and deregulation of apoptosis. For this reason, it was suggested that approaches such as inducing leukemia cell differentiation and apoptosis other than killing cells may be more effective in the treatment of leukemia.

Although a significant progress has been made in the treatment of leukemia, chemotherapy is still the main approach. However, the severe side effects of chemotherapeutic agents, multidrug resistance and relapses during the late stage of treatment limit the effectiveness of these treatments, more and more researchers pay attention to TCM treatment of leukemia. Arsenic trioxide $\left(\mathrm{AS}_{2} \mathrm{O}_{3}, \mathrm{ATO}\right)$, one of the most famous medicines for treating leukemia, it is the main component of white arsenic, a Chinese herbal medicine. It has achieved relatively high remission rate in treating acute promyelocytic leukemia (APL), a type of AML. However, ATO are not effective against all APLs and accompanied by some adverse reactions. APL differentiation syndrome is considered to be the most severe side effect of ATO, with an incidence of 7-35\% [8]. It has also been reported that some patients with ATO treatment may display QT prolongation to $>500 \mathrm{~ms}$ [9]. Furthermore, treatment with 7.5-10 $\mu \mathrm{g} / \mathrm{g}$ ATO resulted in 
hepatic necrosis in animals [10]. Therefore, it is urgent to discover new safe and effective reagents from TCM for leukemia. A. euchroma has a long history to treat blood diseases in TCM. Many studies have shown that shikonin has therapeutic effects on leukemia cells, mainly including HL-60 cells and K562 cells [11-13]. However, the underlying mechanism of action (MoA) for all the ingredients of A. euchroma is barely explored systematically.

With thousands of years of empirical test and refinement, TCM is regarded as a valuable treasure of China. Given the importance of TCM, more and more attentions have been paid on TCM. For complicated or multi-factorial diseases, emerging evidence indicates that TCM has an unexpectedly good effect [14]. In the process of TCM modernization, how to clarify the material basis and MoA of TCM has become the bottleneck of the development of TCM. Since TCM works with multiple components on multiple targets, it is difficult to understand its MoA and material basis of efficacy. In 2007, Dr. Hopkins proposed the concept of network pharmacology [15], which considers the multiple interactions between drugs, targets and diseases [16]. Network pharmacology coincides with the "multi-component, multi-target" characteristics of TCM, and has become a hot spot in the research and development of TCM. There have been many researchers using network pharmacology to study the MoA of TCM. However, in these studies, few ones have paid attentions to the concentrations of various ingredients which could effect on the efficacy of TCM. In this study, we took the concentrations of components in $A$. euchroma into account, and analysed the MoA of A. euchroma on leukemia from the perspective of network pharmacology. 
In recent years, our group has worked on network pharmacology and developed a series of computational methods to predict compound-target interactions (CTIs), including network-based inference (NBI), substructure-drug-target network-based inference (SDTNBI), and balanced SDTNBI (bSDTNBI) [17-19]. Previous studies have demonstrated that these methods could predict potential targets for TCM components reasonably [20-22]. In this study, we used bSDTNBI to predict targets for components of A. euchroma, and then investigated the material basis and MoA of A. euchroma on leukemia via network pharmacology approach.

\section{Methods}

\section{Data collection and preparation}

The components and their concentrations in A. euchroma were collected from several databases including TCM-MESH [23], TCMID [24], Chinese Natural Product Chemical Composition Library (http://pharmdata.ncmi.cn/cnpc/), and Phytochemicals database (http://chemdb.sgst.cn/scdb/main/plant_introduce.asp), as well as related literature from PubMed (https://www.ncbi.nlm.nih.gov/pubmed/), Web of Science (http://apps.webofknowledge.com) and CNKI (http://www.cnki.net/). The structures of these compounds were obtained from NCBI PubChem Database and duplicates were removed.

Known compound-target interactions (KCTIs) for each component of A. euchroma were collected from four databases, including BindingDB [25], IUPHAR/BPS Guide to PHARMACOLOGY [26], ChEMBL [27], and PubChem [28]. Target protein names of 
components were converted into gene names with the species limited into "Homo sapiens" by UniProt (https://www.uniprot.org/). Duplicated KCTIs were removed.

Leukemia related targets were obtained from five databases: OMIM [29], PharmGkb [30], TTD [31], DisGeNET [32], and MalaCards [33].

\section{Prediction of compound-target interactions}

The bSDTNBI method was used to predict potential compound-target interactions (PCTIs) for each component of A. euchroma. In a "substructure-drug-target network", based on the resource diffusion process, bSDTNBI can predict new targets for compounds. In the method, three parameters, namely $\alpha, \beta$ and $\gamma$, that control, respectively, the relative importance of substructure vs. target nodes, drug-substructure vs. drug-target edges, and of hub nodes. The three parameters were optimized by grid search and 10-fold cross validation. First of all, $\alpha$ and $\beta$ were optimized under the condition of ignoring the influence of hub nodes $(\gamma=0)$. We found when $\alpha=0.3$ and $\beta$ $=0.1$, performance of the model can be maximized. Then, under the optimal values of $\alpha$ $=0.3$ and $\beta=0.1$, the optimal value of parameter $\gamma$ was searched. When $\gamma=-0.4$, model performance is the best. Therefore, we set parameters $\alpha=0.3, \beta=0.1, \gamma=-0.4$ and used bSDTNBI method to predict 10 potential targets for each component of A. euchroma. The predicted targets were further standardized as official gene symbols. Eventually, the collected known and predicted CTIs were integrated.

\section{Construction of PPI network and selection of leukemia-related targets}


Targets of components of A. euchroma $\left(\mathrm{T}_{\mathrm{A}}\right)$ and targets related to leukemia $\left(\mathrm{T}_{\mathrm{L}}\right)$ were imported into Cytoscape, respectively. Protein-protein interactions (PPIs) of $\mathrm{T}_{\mathrm{A}}$ and $\mathrm{T}_{\mathrm{L}}$ were obtained by stringAPP, a Cytoscape plugin. High-confidence PPIs with scores above 0.7 were selected to construct A. euchroma PPI network (PPI $\left.{ }_{\mathrm{A}}\right)$ and leukemia PPI network $\left(\mathrm{PPI}_{\mathrm{L}}\right)$. Then $\mathrm{PPI}_{\mathrm{A}}$ and $\mathrm{PPI}_{\mathrm{L}}$ were merged together to construct A. euchromaleukemia PPI network.

A. euchroma-leukemia PPI network was analyzed by CytoNCA, another Cytoscape plugin. Each node in the network has eight central attributes, including subgraph centrality (SC), degree centrality (DC), eigenvector centrality (EC), information centrality (IC), local average connectivity-based method (LAC), betweenness centrality (BC), closeness centrality (CC), and network centrality (NC). For all target nodes, the targets of TA that meet the screening criteria "SC $>$ median $\mathrm{SC} \& \mathrm{DC}>$ median $\mathrm{DC} \&$ $\mathrm{EC}>$ median $\mathrm{EC} \& \mathrm{IC}>$ median $\mathrm{IC} \& \mathrm{LAC}>$ median $\mathrm{LAC} \& \mathrm{BC}>$ median $\mathrm{BC} \& \mathrm{CC}>$ median $\mathrm{CC} \& \mathrm{NC}>$ median $\mathrm{NC}$ " were regarded as leukemia-related targets of $A$. euchroma $\left(\mathrm{T}_{\mathrm{AL}}\right)$ and were retained [34].

\section{Construction of weighted compound-target bipartite network}

Compound-target interactions for $\mathrm{T}_{\mathrm{AL}}$ were obtained from all CTIs (KCTIs + PCTIs). Here the concentrations of components in A. euchroma were used to weight the importance of each component. Since the concentrations of components were determined under different experimental conditions and influenced by many factors, such as the origin and growth time of A. euchroma, we processed the original 
concentration data as follows. Firstly, all the concentrations of components were summarized and standardized to a uniform percentage (mass percentage). Then, the average value of percentage of each component was calculated after the maximum and minimum values were removed. Finally, with "percent concentration value $=0.1 \%$ " as the threshold, components were divided into three classes, and the formula was as following:

$$
\text { Components }\left\{\begin{array}{c}
\text { Class I: weight }=3, \quad \text { concentration }>0.1 \% \\
\text { Class II: weight }=2, \quad 0<\text { concentration }<0.1 \% \\
\text { Class III: weight }=1, \quad \text { without concentration data }
\end{array}\right\}
$$

After all components were weighted, a weighted compound-target bipartite network was constructed via Cytoscape.

\section{Enrichment of KEGG pathways}

KEGG pathway enrichment analysis is usually used to describe the characteristics of query targets. Here, STRING 10.5 (https://string-db.org/cgi/input.pl) [35] was used to perform KEGG pathway enrichment analysis. The KEGG pathways with FDR (False Discovery Rate) $<0.05$ were regarded as significant and useful. Targets in weighted compound-target bipartite network were inputted to STRING, and KEGG pathways with FDR $<0.05$ were obtained. A scoring function was designed to screen pathways, and the equation was as following:

$$
\operatorname{Norm}(Y)=(1-0.1) * \frac{Y_{i}-M I N(Y)}{M A X(Y)-M I N(Y)}+0.1
$$




$$
P S_{m}=\operatorname{Norm}\left(\sum_{j=1}^{j=N_{T}} \operatorname{Norm}\left(\sum_{i=1}^{i=N_{C}} X_{i}\right)\right)+\operatorname{Norm}\left(L F D R_{m}\right)
$$

in which, $P S_{m}$ refers to the score of the m-th KEGG pathway; $N_{T}$ refers to the number of targets involved in the m-th pathway; $N_{C}$ refers to the number of components that interact with the j-th target in the compound-target bipartite network; $X_{i}$ refers to the weight of the i-th component; $\mathrm{LFDR}_{\mathrm{m}}$ refers to the negative logarithmic value of FDR of the $\mathrm{m}$-th pathway; Norm refers to the min-max normalization method with normalize data from 0.1 to 1 .

After all pathways were scored, pathways that meet the role "score $>1.5$ median score" were selected. Referring to the pathway classification standard of KEGG Pathway Database, here, these selected pathways were divided into four categories, including cell survival and death, immune system, endocrine system and specific human diseases. In order to facilitate analysis, we removed specific human disease pathways, which were not directly related to the treatment mechanism of leukemia, and the other pathways were remained.

\section{Selection of hub genes and molecular docking simulation}

Targets that were involved in the above qualified pathways were selected out and scored further by the following scoring function:

$$
\begin{gathered}
\operatorname{Norm}(X)=(1-0.1) * \frac{X_{i}-\operatorname{MIN}(X)}{\operatorname{MAX}(X)-\operatorname{MIN}(X)}+0.1 \\
T S_{j}=N_{p} * \operatorname{Norm}\left(\sum_{i=1}^{i=N_{c}} X_{i}\right)
\end{gathered}
$$


in which, $T S_{j}$ refers to the score of the j-th target; $N_{p}$ refers to the number of pathways that the $\mathrm{j}$-th target involved in; $N_{c}$ refers to the number of components that have interactions with the $\mathrm{j}$-th target; $X_{i}$ refers to the weight of the i-th component; Norm refers to the min-max normalization method with normalize data from 0.1 to 1 .

After all targets were scored, targets that meet "score $>1.5$ median score" were regarded as hub genes, and CTIs that were associated with these targets were selected out. The molecular docking simulation was further performed to validate the binding efficiency of the PCTIs among the selected CTIs. First, we collected the crystal structures of the selected protein from the RCSB Protein Data Bank (PDB, http://www.pdb.org/, updated on 2020-4-10) and selected the relatively higher resolution crystal structures with the ligands. Second, the structures of chemical components contained in A. euchroma were downloaded from the PubChem database (https://pubchem.ncbi.nlm.nih.gov/). Then, protein preparation module of Schrödinger's Maestro Molecular modeling suite (Schrödinger Release 2015-2) was utilized for preparation of the protein crystallographic structures. Water molecules were subsequently deleted from the structures, and the amide moieties in the side chain were adjusted to optimize their interactions with surrounding residues and groups of atoms. Force field OPLS_2005 was also added. A ligand grid generation was based on the ligand in the co-crystallographic structure. The docking region was centered on the ligand, and after the ligand grid was generated, the compounds were imported into Mastero. LigPrep module of the Maestro molecular modeling package was used to obtain the 3D structures and energy minimization of the 
identified compounds. Compounds were docked in the generated grid using the standard Glide docking mode.

\section{Results}

In this study, we took the concentrations of components in A. euchroma into account, and analyzed the MoA of A. euchroma on leukemia by network pharmacology approach, which involved three steps in the whole workflow (Fig. 1): 1) data collection. The components of A. euchroma along with their corresponding targets and leukemia related targets were identified by various databases; (2) network establishment. A. euchromaleukemia PPI network and weighted compound-target bipartite network were constructed via Cytoscape; (3) network analysis. Hub genes were selected out according to KEGG pathways analysis, and molecular docking was used to validate the binding efficiency of PCTIs to construct the critical compound-target bipartite network. Figure 1 depicts a workflow of the technical strategy used in this study.

\section{Components and concentrations of A. euchroma and leukemia related}

\section{targets}

During the process of data collection, a total of 65 components of A. euchroma were obtained, and 27 of them with concentration information (see Supplementary Table S1). Considering that our bSDTNBI method could not tell stereoisomers, components that were mutually stereoisomers were treated as the same molecule among the 65 components. According to the collected concentration information of each component, after the maximum and minimum values were removed, the average of percentage (mass 
percentage) was calculated. Finally, 11 components with concentration greater than $0.1 \%$ had a weight of 3 (Concentration Score, $X=3$ ), 16 components with concentration less than $0.1 \%$ but greater than 0 had a weight of $2(X=2)$, and the remaining 38 components without collected concentration data had a weight of $1(X=1)$.

From OMIM, PharmGkb, TTD, DisGeNET, and MalaCards, 160, 91, 64, 26, and 64 targets related to leukemia were collected, respectively. Totally 332 targets were ultimately reserved after removing the duplicates.

\section{Known and predicted compound-target interactions}

A total of 129 KCTIs for 13 components were collected from databases, which were involved in 92 targets. Only 13 of the 65 components of A. euchroma have known targets. Therefore, it is especially necessary to predict potential targets for components. In this study, 10 targets were predicted for each of the 65 components by our bSDTNBI method. A total of 79 potential targets were obtained, resulting in 650 new CTIs. After the known and predicted CTIs were merged, duplicated CTIs were then removed. Finally, the number of retained CTIs was 779, involved in 65 components and 157 targets (see Supplementary Table S2).

\section{A. euchroma-leukemia PPI network}

157 targets $\left(\mathrm{T}_{\mathrm{A}}=157\right)$ of 65 components in A. euchroma and 332 leukemia related targets $\left(T_{L}=332\right)$ were imported to Cytoscape, respectively. When the confidence (score) cutoff was set to $0.7,129$ targets out of $\mathrm{T}_{\mathrm{A}}$ had 364 PPIs $\left(\mathrm{PPI}_{\mathrm{A}}=364\right)$, while 270 targets out of $\mathrm{T}_{\mathrm{L}}$ had 1335 PPIs $\left(\mathrm{PPI}_{\mathrm{L}}=1335\right)$. When they were merged, 1658 PPIs were 
obtained. Among them, there were 709 PPIs for the 129 targets of A. euchroma components. These 709 PPIs were selected out and re-submitted to Cytoscape to construct A. euchroma-leukemia PPI network. In the PPI network, 8 central attributes were calculated by CytoNCA (see Supplementary Table S3). According to the screening criteria, there were 37 targets of A. euchroma components ( $\mathrm{T}_{\mathrm{AL}}$ ) were qualified (Fig. 2), which corresponding to 55 components and 114 CTIs.

\section{Weighted compound-target bipartite network}

114 CTIs of the $37 \mathrm{~T}_{\mathrm{AL}}$ were imported into Cytoscape to construct the weighted compound-target bipartite network, as shown in Fig. 3 (see Supplementary Table S4). Three weight values were given to each of the components according to their concentrations in A. euchroma. Fig. 3 illustrated the network as a bipartite graph for the components and their potential targets with color-coded nodes. In total, this network consisted of 92 nodes and 114 edges, with 55 components as hexagon nodes and 37 targets as circle nodes. To further clarify the relationships between the active compounds and their targets, we divided the 55 compounds into three groups according to their concentrations, namely Class I components, which have the highest concentration (orange node); Class II components, which have a lower concentration (green node); and Class III components, which do not have concentration information (gray node). There were 10 Class I components, 13 Class II components and 32 Class III components. Among Class I components, only MOL16 (acetylalkannin/acetylshikonin) and MOL18 (alkannin/shikonin) had interactions with more than one target; the remaining ones were associated with only one target. As for Class II components, 8 components had 
interactions with one target, the others were associated with more than one target. NFE2L2 as the target with the most components, it could be hit by 42 components.

\section{Analysis of KEGG pathways}

$37 \mathrm{~T}_{\mathrm{AL}}$ were enriched into $115 \mathrm{KEGG}$ pathways. According to the scoring function, 34 pathways were qualified. We divided the 34 pathways into four categories, including four cell survival and death pathways, two immune system pathways, three endocrine system and 25 specific human diseases pathways (see Supplementary Table S5). For ease of analysis, we remove 25 specific human diseases pathways that are not directly related to possible leukemia treatment mechanisms, and retain other nine pathways. We constructed a pathway-gene network based on the nine pathway-related genes by Cytoscape to identify hub genes in the pathway. The pathway-gene network contained 30 nodes and 53 edges, including 21 genes and 9 pathways (Fig. 4).

There were four cell survival and death pathways include MAPK signaling pathway (hsa04010), PI3K-Akt signaling pathway (hsa04151), Sphingolipid signaling pathway (hsa04071), Apoptosis (hsa04210). These four pathways were related to cell growth, differentiation and apoptosis. MAPK signal transduction pathway plays a crucial role in cell proliferation, differentiation and other processes. As a caspase activator, MAPK also plays an important role on apoptosis. AKT1, CASP3, EGFR, HSPB1, MAPT, TNF, and TP53 participate in the MAPK signaling pathway, and 22 components could act on them. PI3K/Akt signaling pathway is extensively present in cells. It is believed to be one of the pivotal signaling pathways regulating cell growth, proliferation, differentiation and 
apoptosis. Akt (gene symbol as AKT1) is a direct downstream target of PI3K/Akt pathway. Activated Akt can inhibit the release of cytochrome $\mathrm{c}$ from the mitochondria, which blockade the apoptosis triggered by caspases. The PI3K/Akt signaling pathway enriched 8 genes, including AKT1, EGFR, GSK3B, IL2, MCL1, PTEN, TLR2, TP53, and a total of 11 components could act on them. Apoptosis is also known as programmed cell death, which plays a critical role in maintaining development, homeostasis and defending against pathogens. In transmitting apoptotic signals, CASP3 is the major effect factor. The activation of CASP3 triggers apoptosis. MCL1 is a member of the Bcl2 family, which is an apoptotic inhibitor. Inactivation of p53 functions also causes cancer cells to escape apoptosis. The p53 plays a pivotal role in leukemic hematopoiesis. A total of 5 genes were enriched in Apoptosis signaling pathway, they were AKT1, CASP3, MCL1, TNF, TP53, and 15 components were associated with them. Analysis of the above results indicates that induction of cell apoptosis may be a key factor in the treatment of leukemia by A. euchroma.

There were two immune system pathways, include IL-17 signaling pathway (hsa04657) and $\mathrm{T}$ cell receptor signaling pathway (hsa04660). The IL-17 signaling pathway were enriched by five genes, include CASP3, GSK3B, MMP1, MMP9 and TNF. As for T cell receptor signaling pathway (hsa04660), AKT1, FYN, GSK3B, IL2 and TNF were enriched. It's well known that cytokines play a critical role in the context of inflammation, and extensive research has also proved that cytokines exert profound effects on the progression of hematopoietic malignancies including leukemia. It can be 
inferred that A. euchroma relieves inflammatory environment may be related to its treatment of leukemia.

The other three pathways were endocrine system-related pathways, include Estrogen signaling pathway (hsa04915), Thyroid hormone signaling pathway (hsa04919) and Relaxin signaling pathway (hsa04926). A total of 12 genes enriched by these three pathways, they were AKT1, EGFR, ESR1, GSK3B, HDAC3, HIF1A, MMP1, MMP2, MMP9, RARA, SP1 and TP53. Of which, EGFR, one of the transmembrane tyrosine kinase receptors, serves as a stimulus for cancer growth, which is aberrantly activated in most common tumors, including leukemia.

\section{Molecular docking analysis and critical compound-target bipartite network}

According to the scoring function, 10 hub targets were qualified, and 18 components have interactions with them in all CTIs. There were 27 CTIs between the 18 components and 10 targets, and 11 of them were KCTIs, 16 were PCTIs. All of the 16 PCTIs were composed of CTIs related to HIF1A and TNF while all of the CTIs related to HIF1A and TNF were PCTIs. In this study, we used the molecular docking stimulation to identify the binding ability of the 16 pairs of PCTIs that between components of $A$. euchroma and the obtained hub genes. As the above described, only HIF1A and TNFrelated CTIs were predicted, the other hub genes and their associated CTIs were KCTIs. Therefore, the molecular docking simulation was further performed to validate the binding efficiency of HIF1A and TNF with their corresponding components containing 
in A. euchroma. The docking scores of 16 pairs of PCTIs were listed in Table S6. There were 8 pairs of PCTIs had strong binding free energy, since their docking scores were higher than the median value of all pairs. The 8 pairs of PCTIs consisted of 6 pairs of TNF-related PCTIs and 2 of HIF1A. The action modes between TNF and its corresponding compounds were shown in Fig.5a-f, the interplay between HIF1A and its corresponding compounds were shown in Fig. $5 \mathrm{~g}$-h. It can be found that dehydroalkannin (MOL24) could form a hydrogen bond with Tyr151, oleanolic acid (MOL47) could form a hydrogen bond with Ser60. In addition, dehydroalkannin (MOL24) could form a $\pi-\pi$ interaction with Tyr59. Besides, arnebinone (MOL6) had the best binding ability with TNF (docking score $=-6.222$ ), and followed by arnebiabinone (MOL21) (docking score $=-5.658)$, dehydroalkannin (MOL24) (docking score $=-5.405)$. Finally, the 8 pairs of PCTIs and 11 pairs of KCTIs were used to critical compound-target bipartite network, which involved in 10 targets and 14 components, as shown in Fig. 6. Here, 2 (MOL6 and MOL8) components were monoterpene phenol and benzoquinones (green nodes), 4 (MOL24, MOL21, MOL18 and MOL16) were naphthoquinones (gold node), 3 (MOL65, MOL47 and MOL49) were triterpenoids (blue nodes), 1 (MOL41) was alkaloids (cyan node), 2 (MOL38 and MOL36) were steroids (purple nodes), and the other 2 (MOL42 and MOL53) were organic acids (brown nodes). Structures of the 14 components were shown in Supplementary Figure S2.

\section{Discussion}

A. euchroma is famous for its effect of heat cleaning and blood cooling. In recent years, more and more studies have indicated that shikonin and some of its derivatives can 
inhibit leukemia cell proliferation, induce leukemia cell differentiation and promote apoptosis. Most of the studies focus on individual or several ingredients, and the effects of all components of A. euchroma have never been explored systematically. Moreover, although the importance of TCM dosage has received widespread attention, few TCMrelated network pharmacology studies have considered the influence of ingredient concentration on the effectiveness of TCM. In this study, we employed network pharmacology approach to further explore the MoA of A. euchroma on leukemia. We retrieved 65 components and 157 targets of A. euchroma from known databases and literature. Combined with network analysis and molecular docking simulation, we found that 14 components and 10 targets were potential critical components and targets, which might contribute to the effect of A. euchroma on leukemia treatment. Our results indicated that the possible MoA would mainly involve in three aspects: 1 . A. euchroma inhibits leukemia cell survival and induces apoptosis; 2. A. euchroma relieves inflammatory environment; 3. A. euchroma inhibits angiogenesis.

Apoptosis, also known as programmed cell death, is a physiological process or some pathological condition, in which the cells take part in the death process after they are triggered by a certain signal. Inhibiting leukemia cell survival and inducing leukemia cell apoptosis is a promising therapeutic approach for leukemia. There were 4 pathways (MAPK signaling pathway (hsa04010), PI3K-Akt signaling pathway (hsa04151), Sphingolipid signaling pathway (hsa04071), Apoptosis (hsa04210)) were related to cell survival and apoptosis and involved in 13 targets, 26 components in weighted compound-target bipartite network. In transmitting apoptotic signals, CASP3 is the 
major effect factor. The activation of CASP3 triggers apoptosis. As one of the caspase activators, MAPK is well known to play a crucial role in apoptosis. Extensive studies have shown that targeting to MAPK signaling cascades, alone or in combination with other drugs, results in enhanced anti-leukemic responses in AML [36,37]. It has been shown that activation of p38 MAPK promoted BCL2 degradation, which partly induced K562 cell apoptosis [38]. Huang et al. reported that shikonin treatment activated p38 MAPK and JNK phosphorylation in human lens epithelial cells [39]. The PI3K/Akt signaling pathway regulates diverse cellular processes, including growth, proliferation, differentiation and apoptosis by phosphorylating its downstream target, including BCL2L11, BAD, MDM2, XIAP, CASP9, GSK3B and so on. TP53 is a tumor suppressor protein and regulator of the cell cycle, which plays a pivotal role in leukemic hematopoiesis, but its functions are frequently suppressed by MDM2. Therefore, inhibition of MDM2 is beneficial to the stability of TP53. However, Akt enhances MDM2 activity, which is harmful for the stability of TP53. PTEN is a main negative regulator of the PI3K/Akt pathway, and plays an important role in maintaining hematopoietic stem cells and preventing leukemia [40]. It has been demonstrated that impaired PTEN function and aberrantly activated PI3K/Akt are present in many leukemia cases [11,41]. Pan et al. suggested that combined BCL2 inhibition and TP53 activation may be a promising therapeutic approach for AML [42].

It is well known that inflammation is one of the major barriers to cancer therapy. In recent years, it has become evident that inflammation plays a key role in leukemia [43]. As a pro-inflammatory cytokine, TNF is recognized as a key mediator of inflammatory 
reactions in tumor tissues, and also responsible for increased NF- $\kappa \mathrm{B}$ activity in many tumors. TNF promotes the progression of several hematopoietic tumors by participating in several signaling pathways, including NF- $\kappa \mathrm{B}$, and PI3K/Akt $[44,45]$. It has been reported that TNF can be produced by various leukemia cells, including AML [36], ALL [46], CML [47], CLL [48], and so on. There was also evidence showing that TNF induced MMP9 expression or secretion in leukemia cells [49]. TNF has been used as an anti-cancer drugs in various cancer cells. Staniforth et al. found that shikonin (MOL18), isobutyrylshikonin (MOL26), acetylshikonin (MOL16), beta,betadimethylacrylshikonin (MOL31) and isovalerylshikonin (MOL27) showed significant dose-dependent inhibition of TNF in vivo [50].

Angiogenesis is a critical element to cancer cell survival; increased vascularity was found in patients with acute myeloid leukemia [51]. HIF1A, a key regulator of the cellular response to hypoxia, which controls a vast array of gene products involved in energy metabolism, glycolysis, angiogenesis, apoptosis and cell cycle [52]. HIF1A has been recognized as a strong promoter of tumor growth and it is responsible for VEGF gene expression [53]. VEGF is one of the major angiogenesis-activating protein, it has been implicated in leukemia-associated angiogenesis [54]. There were some data showed that HIF1A over-expressed in some leukemia cells $[55,56]$. Frolova et al. reported that blockade of HIF1A-mediated signaling may enhance the efficacy of the therapeutic regiments in acute lymphocytic leukemia [57]. Matrix metalloproteinase (MMPs) were found highly expressed in B-CLL cells, and promoted the migration and invasion of leukemia cells [58]. MMP9, secreted by leukemic cells, mediate opening of the blood-brain barrier by disrupting tight junction proteins in CNS leukemia [59]. 
Sustained or enhanced MMP9 secretion plays an important role in the pathophysiology of tumor progression.

According to the above analysis, we selected 10 targets and 14 components as critical targets and key components. Among them, shikonin (MOL18) was the most studied components of A. euchroma, it was demonstrated to have anti-inflammatory and proapoptotic effects [12,60]. Acetylshikonin (MOL16) was found induced tumor cell apoptosis through activating the pro-apoptotic bcl-2 family and caspase-3 [61].

As one of the most famous Chinese medicine for treating leukemia, ATO is an effective and relatively safe drug in the treatment of APL. Combining compound-target network of A. euchroma and related literature, we compared ATO and A. euchroma in terms of leukemia treatment, and we found some commonalities: (1) MAPK signaling pathway. ATO has been demonstrated to activate all three MAPKs pathways, ERKs, JNKs, and p38 kinases to exert its anti-leukemia effect [62]. The MAPK signaling pathway was also included in pathways that enriched by targets of A. euchroma. (2) Angiogenesis. ATO was reported to inhibit angiogenesis by inhibiting leukemic cell VEGF production [63]. As for A. euchroma, 7 components could act on HIF1A, which is responsible for increased expression of VEGF. Moreover, several studies have shown that some components of A. euchroma exhibited antiangiogenic activity by inhibiting VEGF [64,65]. (3) Caspases. ATO was shown to increase caspases activity to induce apoptosis of leukemia cells [66]. Extensive studies have demonstrated that components of $A$. euchroma increased the expression of caspases to induce apoptosis [12,67]. As a result, 
we have reasons to speculate that A. euchroma may be a potential anti-leukemia agent with less toxic than ATO.

In this study, we found that the MoA of A. euchroma on leukemia involves the inhibition of leukemia cell survival and induction of leukemia cell apoptosis, the relief of inflammatory environment, and the inhibition of angiogenesis. Concretely speaking, it includes three aspects: (1) A. euchroma impacts the PI3K/AKT and MAPK signaling pathway, which inhibit leukemia cell survival and induce leukemia cell apoptosis. (2) A. euchroma inhibits the expression of inflammatory medium, such as TNF, IL6, IL2 and so on to relieve inflammatory environment. (3) A. euchroma inhibits angiogenesis by regulating HIF1A, MMP2, MMP9 and other factors related to angiogenesis. The results preliminarily validated and explained the therapeutic material basis and mechanism of A. euchroma on leukemia, which provided a preliminary information and basis for further in-depth exploration of its MoA, and a reference for the study of the more complex components of TCM.

\section{Conclusions}

A. euchroma, a Chinese herb with the effects of heat cleaning and blood cooling, has been used to treat blood diseases in TCM for thousands of years. In this work, we investigated the interactions between chemical components of A. euchroma and related targets involved in the MoA by network pharmacology approaches. Considering the concentration information of components in A. euchroma and combined with the methods of network analysis and molecular docking simulation, we found that 55 active 
components and 37 targets might related to the anti-leukemia effects of A. euchroma, of which 14 components and 10 targets were considered to be critical. It was demonstrated that A. euchroma has potential anti-leukemia activities because of its effects on inhibiting leukemia cell survival and inducing apoptosis, relieving inflammatory environment and inhibiting angiogenesis. We further realized that, not only shikonin, the most fully studied ingredient of A. euchroma, one of compound of naphthoquinones, other components among the 14 critical components of A. euchroma, including monoterpene phenol and benzoquinones, triterpenoids, alkaloids, steroids and organic acids would also have therapeutic effects on leukemia.

In summary, we applied network pharmacology approach to study the multi-component, multi-target networks involved in the treatment effect of A. euchroma on leukemia. The results preliminarily validated and explained the therapeutic material basis and mechanism of A. euchroma on leukemia, which provided a preliminary information and basis for further in-depth exploration of its MoA, and a reference for the study of the more complex components of TCM.

\section{Abbreviations}

\section{A. euchroma: Arnebia euchroma}

TCM: traditional Chinese medicine

MoA: mechanism of action

CTIs: compound-target interactions 
bSDTNBI: balanced substructure-drug-target network-based inference

PPIs: protein-protein interactions

KCTIs: known compound-target interactions

PCTIs: predicted compound-target interactions

AML: acute myeloid leukemia

ALL: acute lymphoblastic leukemia

CML: chronic myeloid leukemia

CLL: chronic lymphoblastic leukemia

ATO: Arsenic trioxide

APL: acute promyelocytic leukemia

NBI: network-based inference

SDTNBI: substructure-drug-target network-based inference

$\mathbf{T}_{\mathbf{A}}$ : Targets of components of A. euchroma

$\mathbf{T}_{\mathbf{L}}$ : targets related to leukemia

$\mathbf{P P I}_{\mathbf{A}}$ : A. euchroma PPI network 
PPI $_{\text {L }}$ leukemia PPI network

SC: subgraph centrality

DC: degree centrality

EC: eigenvector centrality

IC: Information centrality

LAC: local average connectivity-based method

BC: betweenness centrality

CC: closeness centrality

NC: network centrality

$\mathbf{T}_{\mathrm{AL}}$ : leukemia-related targets of A. euchroma

FDR: False Discovery Rate

PDB: RCSB Protein Data Bank

MMPs: Matrix metalloproteinase

\section{Funding}

This work was supported by the National Natural Science Foundation of China (Grants

U1603122 and 81673356) and the 111 Project (Grant BP0719034). 


\section{Author contributions}

YT, JW, BZ and BW contributed to conception and design of the study. BW performed the experiments and wrote the manuscript. ZW provided the method of target prediction. WL and GL contributed to the writing of this article. YT implemented the study and modified the manuscript. All authors have discussed the results and approved the final manuscript.

\section{Acknowledgments}

This is a short text to acknowledge the contributions of specific colleagues, institutions, or agencies that aided the efforts of the authors.

\section{Competing interests}

The authors declare that they have no competing interests.

\section{Availability of data and materials}

The data can be requested from the author.

\section{Consent for publication}

Not applicable.

\section{Ethics approval and consent to participate}

Not applicable.

\section{Author details}

${ }^{1}$ Shanghai Key Laboratory of New Drug Design, School of Pharmacy, East China University of Science and Technology, Shanghai 200237, China. 
${ }^{2}$ Key Laboratory of Xinjiang Phytomedicine Resource and Utilization, Ministry of Education, School of Pharmacy, Shihezi University, Shihezi 832002, China.

\section{Corresponding author}

Correspondence to Yun Tang.

\section{Supplementary information}

Additional file 1: Figure S1. Two configurations of naphthoquinones. Figure S2. Structures of the 14 critical components. Table S1. Components of A. euchroma and content score. Table S2. Known and predicted component-target interactions. Table S3. Eight central attributes of targets. Table S4. Weighted component-important target interactions. Table S5. 34 KEGG pathways. Table S6. Docking scores of 16 pairs of PCTIs.

\section{References}

1. Andujar I, Rios JL, Giner RM, Recio MC. Pharmacological properties of shikonin - a review of literature since 2002. Planta Med. 2013/10/25. 2013;79:1685-97. Available from: https://www.thiemeconnect.de/products/ejournals/pdf/10.1055/s-0033-1350934.pdf

2. Chen X, Yang L, Oppenheim JJ, Howard MZ. Cellular pharmacology studies of shikonin derivatives. Phytother Res. 2002;16:199-209. Available from:

http://www.ncbi.nlm.nih.gov/pubmed/12164262

3. Chen X, Yang L, Zhang N, Turpin JA, Buckheit RW, Osterling C, et al. Shikonin, a component of chinese herbal medicine, inhibits chemokine receptor function and suppresses human immunodeficiency virus type 1. Antimicrob Agents Chemother. 2003;47:2810-6. Available from: 
http://www.ncbi.nlm.nih.gov/pubmed/12936978

4. Wang RB, Yin RT, Zhou W, Xu DF, Li SS. Shikonin and its derivatives: a patent review. Expert Opin Ther Pat. 2012;22:977-97.

5. Papageorgiou VP, Assimopoulou AN, Couladouros EA, Hepworth D, Nicolaou KC. The chemistry and biology of alkannin, shikonin, and related naphthazarin natural products. Angew Chemie Int Ed. 1999;38:270-300.

6. Hu Y, Jiang Z, Leung KSY, Zhao Z. Simultaneous determination of naphthoquinone derivatives in Boraginaceous herbs by high-performance liquid chromatography. Anal Chim Acta. 2006;577:26-31. 7. Zan K, Zheng HY, Liu J, Guo LN, Zheng J, Ma SC. Simultaneous determination of eight hydroxyl naphthoquinones in different parts of Arnebiae euchroma by HPLC. Zhongguo Zhong Yao Za Zhi. 2017;42:2532-7. Available from: http://www.ncbi.nlm.nih.gov/pubmed/28840695

8. Lengfelder E, Hofmann WK, Nowak D. Impact of arsenic trioxide in the treatment of acute promyelocytic leukemia. Leukemia. Nature Publishing Group; 2012;26:433-42. Available from: http://dx.doi.org/10.1038/leu.2011.245

9. Iland HJ, Bradstock K, Supple SG, Catalano A, Collins M, Hertzberg M, et al. All-trans-retinoic acid, idarubicin, and IV arsenic trioxide as initial therapy in acute promyelocytic leukemia (APML4). Blood. 2012;120:1570-80.

10. Rego EM, He LZ, Warrell RP, Wang ZG, Pandolfi PP. Retinoic acid (RA) and As2O3 treatment in transgenic models of acute promyelocytic leukemia (APL) unravel the distinct nature of the leukemogenic process induced by the PML-RARa and PLZF-RAR $\alpha$ oncoproteins. Proc Natl Acad Sci U S A. 2000;97:10173-8.

11. Chen Y, Wang T, Du J, Li Y, Wang X, Zhou Y, et al. The critical role of PTEN/PI3K/AKT 
signaling pathway in shikonin-induced apoptosis and proliferation inhibition of chronic myeloid leukemia. Cell Physiol Biochem. 2018;47:981-93.

12. Mao X, Yu CR, Li WH, Li WX. Induction of apoptosis by shikonin through a ROS/JNK-mediated process in Bcr/Abl-positive chronic myelogenous leukemia (CML) cells. Cell Res. 2008;18:879-88.

13. Zhang B, Chen N, Chen HM, Wang ZH, Zheng QS. The Critical Role of Redox Homeostasis in Shikonin-Induced HL-60 Cell Differentiation via Unique Modulation of the Nrf2/ARE Pathway. Oxid Med Cell Longev. 2012;2012.

14. Zhang J, He Y, Jiang X, Jiang H, Shen J. Nature brings new avenues to the therapy of central nervous system diseases - An overview of possible treatments derived from natural products. Sci China Life Sci. 2019;62:1332-1367.

15. Hopkins AL. Network Pharmacology. Nat Biotechnol. 2007;25:1110-1111.

16. Hopkins AL. Network pharmacology: The next paradigm in drug discovery. Nat Chem Biol. 2008;4:682-90.

17. Cheng F, Liu C, Jiang J, Lu W, Li W, Liu G, et al. Prediction of drug-target interactions and drug repositioning via network-based inference. PLoS Comput Biol. 2012;8:1-12.

18. Wu Z, Lu W, Wu D, Luo A, Bian H, Li J, et al. In silico prediction of chemical mechanism of action via an improved network-based inference method. Br J Pharmacol. 2016;173:3372-85.

19. Wu Z, Cheng F, Li J, Li W, Liu G, Tang Y. SDTNBI: An integrated network and chemoinformatics tool for systematic prediction of drug-target interactions and drug repositioning. Brief Bioinform. 2017;18:333-47.

20. Xiao X, Chen Z, Wu Z, Wang T, Li W, Liu G, et al. Insights into the antineoplastic mechanism of Chelidonium majus via systems pharmacology approach. Quant Biol. 2019;1-12. 
21. Wang T, Wu Z, Sun L, Li W, Liu G, Tang Y. A computational systems pharmacology approach to investigate molecular mechanisms of herbal formula Tian-Ma-Gou-Teng-Yin for treatment of Alzheimer's disease. Front Pharmacol. 2018;9:1-16.

22. Wang YY, Li J, Wu ZR, Zhang B, Yang H Bin, Wang Q, et al. Insights into the molecular mechanisms of Polygonum multiflorum Thunb-induced liver injury: A computational systems toxicology approach. Acta Pharmacol Sin. Nature Publishing Group; 2017;38:719-32. Available from: http://dx.doi.org/10.1038/aps.2016.147

23. Zhang RZ, Yu SJ, Bai H, Ning K. TCM-Mesh: The database and analytical system for network pharmacology analysis for TCM preparations. Sci Rep. Springer US; 2017;7:1-14.

24. Xue R, Fang Z, Zhang M, Yi Z, Wen C, Shi T. TCMID: Traditional Chinese medicine integrative database for herb molecular mechanism analysis. Nucleic Acids Res. 2013;41:1089-95.

25. Gilson MK, Liu T, Baitaluk M, Nicola G, Hwang L, Chong J. BindingDB in 2015: A public database for medicinal chemistry, computational chemistry and systems pharmacology. Nucleic Acids Res. 2016;44:D1045-53.

26. Harding SD, Sharman JL, Faccenda E, Southan C, Pawson AJ, Ireland S, et al. The IUPHAR/BPS Guide to PHARMACOLOGY in 2018: Updates and expansion to encompass the new guide to IMMUNOPHARMACOLOGY. Nucleic Acids Res. Oxford University Press; 2018;46:D1091-106.

27. Gaulton A, Hersey A, Nowotka ML, Patricia Bento A, Chambers J, Mendez D, et al. The ChEMBL database in 2017. Nucleic Acids Res. 2017;45:D945-54.

28. Wang Y, Xiao J, Suzek TO, Zhang J, Wang J, Bryant SH. PubChem: A public information system for analyzing bioactivities of small molecules. Nucleic Acids Res. 2009;37:623-33.

29. Amberger J, Bocchini CA, Scott AF, Hamosh A. McKusick’s Online Mendelian Inheritance in 
Man (OMIM(R)). Nucleic Acids Res. 2009;37:D793-6. Available from:

https://academic.oup.com/nar/article-lookup/doi/10.1093/nar/gkn665

30. Whirl-Carrillo M, McDonagh EM, Hebert JM, Gong L, Sangkuhl K, Thorn CF, et al.

Pharmacogenomics knowledge for personalized medicine. Clin Pharmacol Ther. 2012;92:414-7.

31. Li YH, Yu CY, Li XX, Zhang P, Tang J, Yang Q, et al. Therapeutic target database update 2018:

Enriched resource for facilitating bench-to-clinic research of targeted therapeutics. Nucleic Acids Res.

Oxford University Press; 2018;46:D1121-7.

32. Piñero J, Bravo Á, Queralt-Rosinach N, Gutiérrez-Sacristán A, Deu-Pons J, Centeno E, et al.

DisGeNET: A comprehensive platform integrating information on human disease-associated genes and variants. Nucleic Acids Res. 2017;45:D833-9.

33. Rappaport N, Twik M, Plaschkes I, Nudel R, Stein TI, Levitt J, et al. MalaCards: An amalgamated human disease compendium with diverse clinical and genetic annotation and structured search. Nucleic Acids Res. 2017;45:D877-87.

34. Song W, Ni S, Fu Y, Wang Y. Uncovering the mechanism of Maxing Ganshi Decoction on asthma from a systematic perspective: A network pharmacology study. Sci Rep. 2018;8:1-11.

35. Szklarczyk D, Franceschini A, Wyder S, Forslund K, Heller D, Huerta-Cepas J, et al. STRING v10: Protein-protein interaction networks, integrated over the tree of life. Nucleic Acids Res.

2015;43:D447-52.

36. Volk A, Li J, Xin J, You D, Zhang J, Liu X, et al. Co-inhibition of NF- $\kappa B$ and JNK is synergistic in TNF-expressing human AML. J Exp Med. 2014;211:1093-108. Available from: http://www.jem.org/lookup/doi/10.1084/jem.20130990

37. Geest CR, Coffer PJ. MAPK signaling pathways in the regulation of hematopoiesis. J Leukoc Biol. 
2009;86:237-50.

38. Changchien JJ, Chen YJ, Huang CH, Cheng TL, Lin SR, Chang L Sen. Quinacrine induces apoptosis in human leukemia K562 cells via p38 MAPK-elicited BCL2 down-regulation and suppression of ERK/c-Jun-mediated BCL2L1 expression. Toxicol Appl Pharmacol. Elsevier Inc.;

2015;284:33-41. Available from: http://dx.doi.org/10.1016/j.taap.2015.02.005

39. Huang WR, Zhang Y, Tang X. Shikonin inhibits the proliferation of human lens epithelial cells by inducing apoptosis through ROS and caspase-dependent pathway. Molecules. 2014/06/26.

2014;19:7785-97. Available from: http://www.mdpi.com/1420-3049/19/6/7785/pdf

40. Zhang J, Grindley JC, Yin T, Jayasinghe S, He XC, Ross JT, et al. PTEN maintains haematopoietic stem cells and acts in lineage choice and leukaemia prevention. Nature. 2006;441:518-22.

41. Lamy T, Moignet A, Loughran TP. LGL leukemia: From pathogenesis to treatment. Blood.

2017;129:1082-94.

42. Pan R, Ruvolo V, Mu H, Leverson JD, Nichols G, Reed JC, et al. Synthetic Lethality of Combined Bcl-2 Inhibition and p53 Activation in AML: Mechanisms and Superior Antileukemic Efficacy. Cancer Cell. Elsevier Inc.; 2017;32:748-760.e6. Available from: https://doi.org/10.1016/j.ccell.2017.11.003

43. Binder S, Luciano M, Horejs-hoeck J. The cytokine network in acute myeloid leukemia ( AML ): A focus on pro- and anti-inflammatory mediators. Cytokine Growth Factor Rev. Elsevier; 2018;43:8-15. Available from: https://doi.org/10.1016/j.cytogfr.2018.08.004

44. Gu L, Findley HW, Zhu N, Zhou M. Endogenous TNF $\alpha$ mediates cell survival and chemotherapy resistance by activating the PI3K/Akt pathway in acute lymphoblastic leukemia cells. Leukemia. 2006;20:900-4.

45. Tsai HJ, Kobayashi S, Izawa K, Ishida T, Watanabe T, Umezawa K, et al. Bioimaging analysis of 
nuclear factor-kB activity in Philadelphia chromosome-positive acute lymphoblastic leukemia cells reveals its synergistic upregulation by tumor necrosis factor- $\alpha$-stimulated changes to the microenvironment. Cancer Sci. 2011;102:2014-21.

46. Potapnev MP, Petyovka N V., Belevtsev M V., Savitskiy VP, Migal N V. Plasma level of tumor necrosis factor-alpha (TNF- $\alpha$ ) correlates with leukocytosis and biological features of leukemic cells, but not treatment response of children with acute lymphoblastic leukemia. Leuk Lymphoma.

2003;44:1077-9.

47. Gallipoli P, Pellicano F, Morrison H, Laidlaw K, Allan EK, Bhatia R, et al. Autocrine TNF- $\alpha$ production supports CML stem and progenitor cell survival and enhances their proliferation. Blood. 2013;122:3335-9.

48. Lech-Maranda E, Grzybowska-Izydorczyk O, Wyka K, Mlynarski W, Borowiec M, Antosik K, et al. Serum tumor necrosis factor- $\alpha$ and interleukin-10 levels as markers to predict outcome of patients with chronic lymphocytic leukemia in different risk groups defined by the IGHV mutation status. Arch Immunol Ther Exp (Warsz). 2012;60:477-86.

49. Heidinger M, Kolb H, Krell HW, Jochum M, Ries C. Modulation of autocrine TNF- $\alpha$-stimulated matrix metalloproteinase 9 (MMP-9) expression by mitogen-activated protein kinases in THP-1 monocytic cells. Biol Chem. 2006;387:69-78.

50. Staniforth V, Wang SY, Shyur LF, Yang NS. Shikonins, Phytocompounds from Lithospermum erythrorhizon, Inhibit the Transcriptional Activation of Human Tumor Necrosis Factor $\alpha$ Promoter in Vivo. J Biol Chem. 2004;279:5877-85.

51. Padro T, Ruiz S, Bieker R, Bu H, Steins M, Kienast J, et al. Increased angiogenesis in the bone marrow of patients with acute myeloid leukemia. 2019;95:2637-45. 
52. Semenza GL. Hypoxia-Inducible Factor 1 and Cardiovascular Disease. Annu Rev Physiol. $2014 ; 76: 39-56$.

53. Forsythe JA, Jiang BH, Iyer NV, Agani F, Leung SW, Koos RD, et al. Activation of vascular endothelial growth factor gene transcription by hypoxia-inducible factor 1. Mol Cell Biol.

1996;16:4604-13.

54. Bellamy WT, Richter L, Sirjani D, Roxas C, Glinsmann-Gibson B, Frutiger Y, et al. Vascular endothelial cell growth factor is an autocrine promoter of abnormal localized immature myeloid precursors and leukemia progenitor formation in myelodysplastic syndromes. Blood. 2001;97:1427-34. 55. Matthias Mayerhofer, Peter Valent, Wolfgang R. Sperr, James D. Griffin and CS. BCR/ABL induces expression of vascular endothelial growth factor and its. 2016;51:278-85.

56. Wang Y, Liu Y, Malek SN, Zheng P, Liu Y. Targeting HIF1 $\alpha$ eliminates cancer stem cells in hematological malignancies. Cell Stem Cell. 2011;8:399-411.

57. Forsythe JA, Jiang BH, Iyer NV, Agani F, Leung SW, Koos RD, et al. Regulation of HIF-1 $\alpha$ signaling and chemoresistance in acute lymphocytic leukemia under hypoxic conditions of the bone marrow microenvironment. Cancer Biol Ther. 2012;13:858-70.

58. Redondo-muñoz J, Escobar-díaz E, Samaniego R, Terol MJ, José A, Dc W, et al. MMP-9 in B-cell chronic lymphocytic leukemia is up-regulated by $\alpha 4 \beta 1$ integrin or CXCR4 engagement via distinct signaling pathways, localizes to podosomes, and is involved in cell invasion and migration. Blood. 2006;108:3143-51.

59. Feng S, Cen J, Huang Y, Shen H, Yao L, Wang Y, et al. Matrix metalloproteinase-2 and -9 secreted by leukemic cells increase the permeability of blood-brain barrier by disrupting tight junction proteins. PLoS One. 2011;6:e20599. 
60. Yang C, Liu P, Wang S, Zhao G, Zhang T, Guo S, et al. Shikonin exerts anti-inflammatory effects in LPS-induced mastitis by inhibiting NF- $\kappa \mathrm{B}$ signaling pathway. Biochem Biophys Res Commun. Elsevier Ltd; 2018;505:1-6. Available from: https://doi.org/10.1016/j.bbrc.2018.08.198 61. Xiong W, Luo G, Zhou L, Zeng Y, Yang W. In vitro and in vivo antitumor effects of acetylshikonin isolated from Arnebia euchroma (Royle) Johnst (Ruanzicao) cell suspension cultures. Chin Med. 2009;4:1-7.

62. Bode AM, Dong Z. The paradox of arsenic: Molecular mechanisms of cell transformation and chemotherapeutic effects. Crit Rev Oncol Hematol. 2002;42:5-24.

63. Roboz GJ, Dias S, Lam G, Lane WJ, Soignet SL, Warrell J, et al. Arsenic trioxide induces doseand time-dependent apoptosis of endothelium and may exert an antileukemic effect via inhibition of angiogenesis. Blood. 2000;96:1525-30.

64. Lee H, Lee H, Magesh V, Nam D, Lee E, Ahn KS, et al. Shikonin, acetylshikonin, and isobutyroylshikonin inhibit VEGF-induced angiogenesis and suppress tumor growth in lewis lung carcinoma-bearing mice. Yakugaku Zasshi. 2008;128:1681-8. Available from: http://www.scopus.com/inward/record.url?eid=2-s2.0-56049098575\&partnerID=tZOtx3y1 65. Xu Y, Xu X, Gao X, Chen H, Geng L. Shikonin suppresses IL-17-induced VEGF expression via blockage of JAK2/STAT3 pathway. Int Immunopharmacol. Elsevier B.V.; 2014;19:327-33. Available from: http://dx.doi.org/10.1016/j.intimp.2014.01.027

66. Perkins C, Kim CN, Fang G, Bhalla KN. Arsenic induces apoptosis of multidrug-resistant human myeloid leukemia cells thin express Bcr-Abl or overexpress MDR, MRP, Bcl-2, or Bcl-x(L). Blood. 2000;95:1014-22.

67. Xiong Y, Ma XY, Zhang Z, Shao ZJ, Zhang YY, Zhou LM. Apoptosis induced by beta,beta- 
dimethylacrylshikonin is associated with Bcl-2 and NF-kappaB in human breast carcinoma MCF-7

cells. Oncol Lett. 2013/11/22. 2013;6:1789-93. Available from: https://www.spandidos-

publications.com/ol/6/6/1789/download 


\section{Figures}

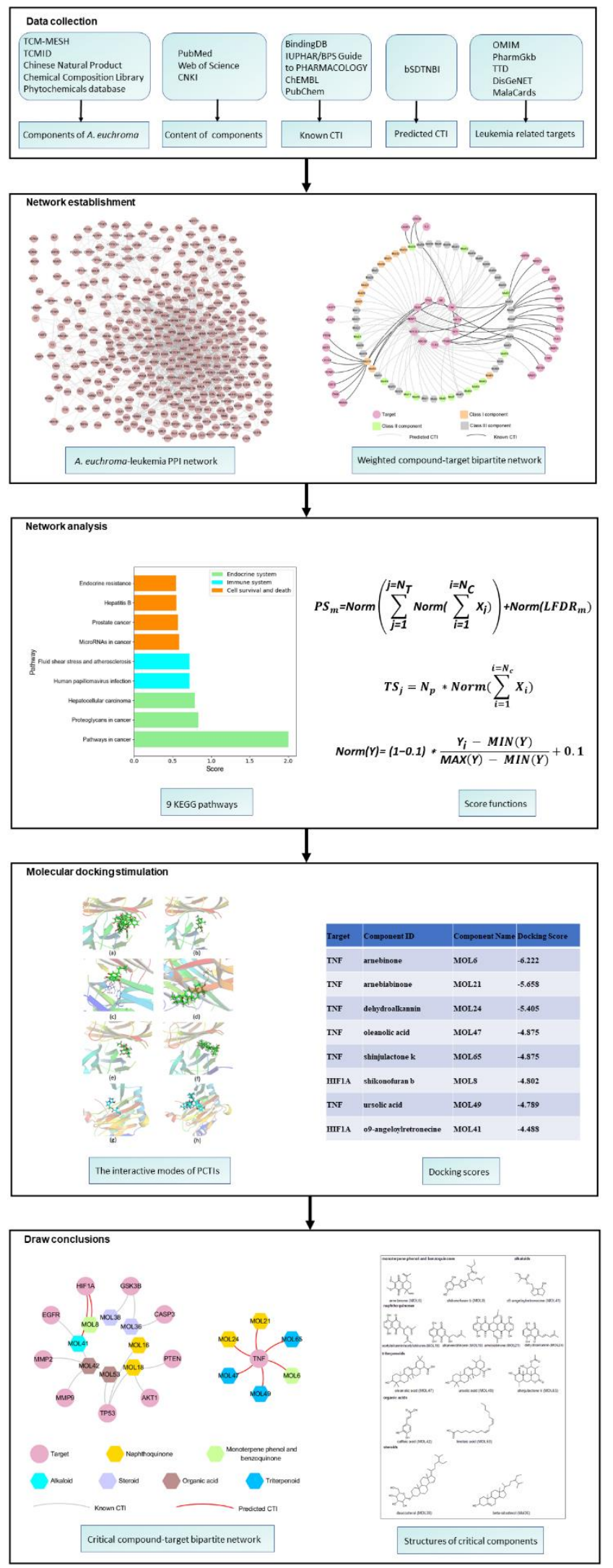

Figure 1. Workflow for A. euchroma on leukemia. 

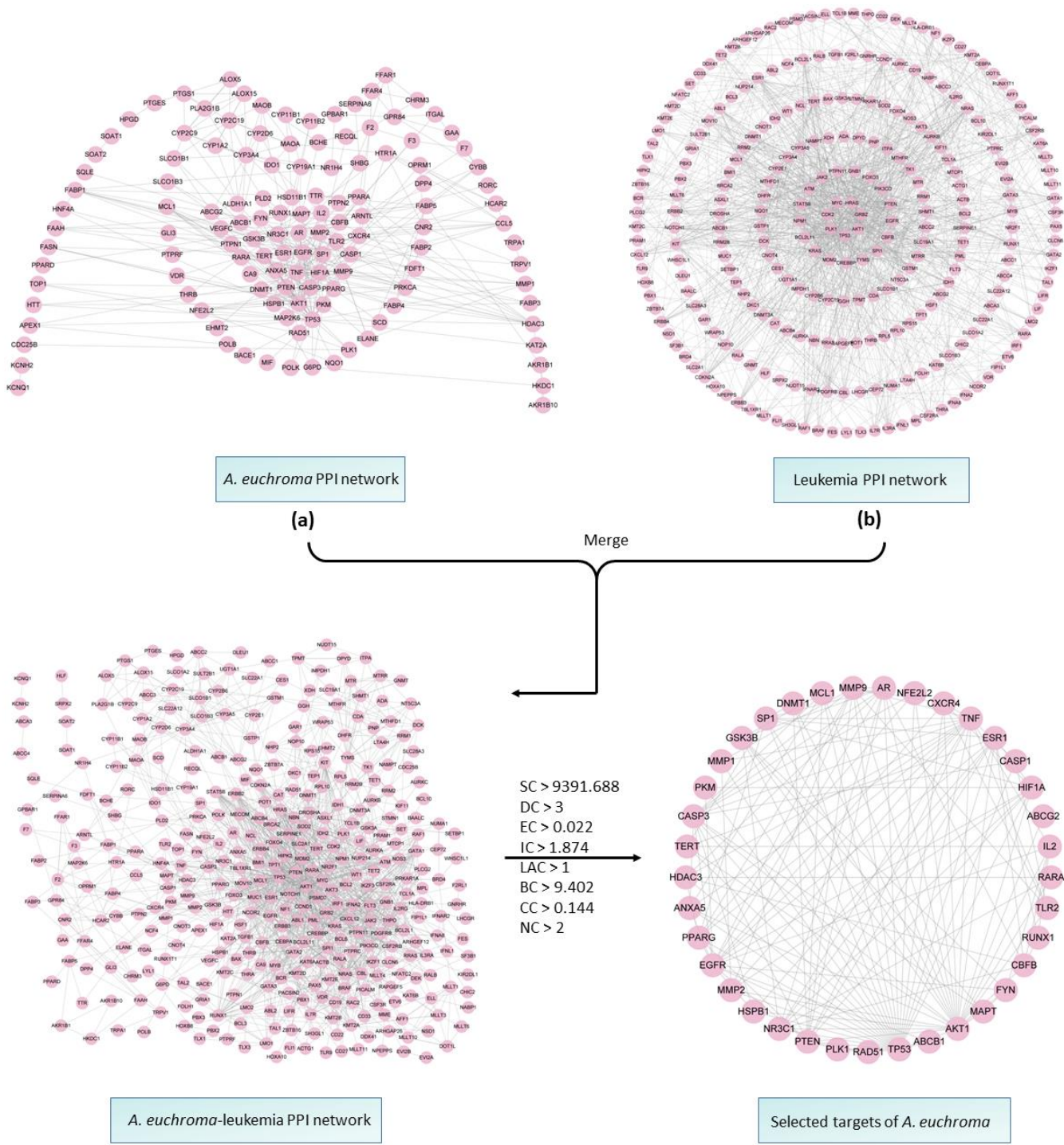

(c)

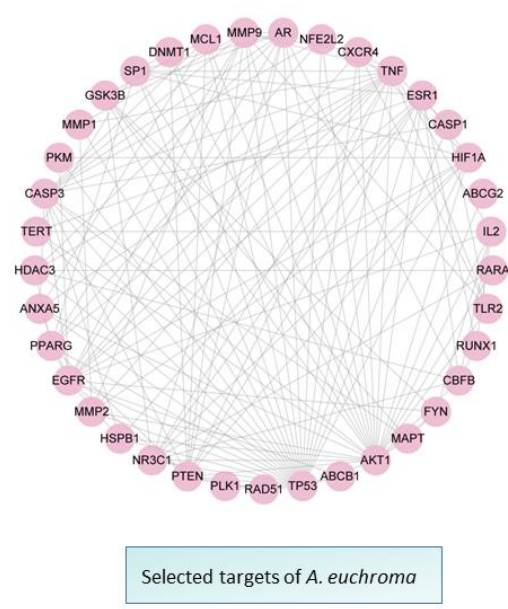

(d)

Figure 2. Construction of A. euchroma-leukemia PPI network. (a) A. euchroma PPI network (PPIA); (B) Leukemia PPI network (PPIL); (C) A. euchroma-leukemia PPI network; (D) Targets of A. euchroma that meet the screening criteria. 


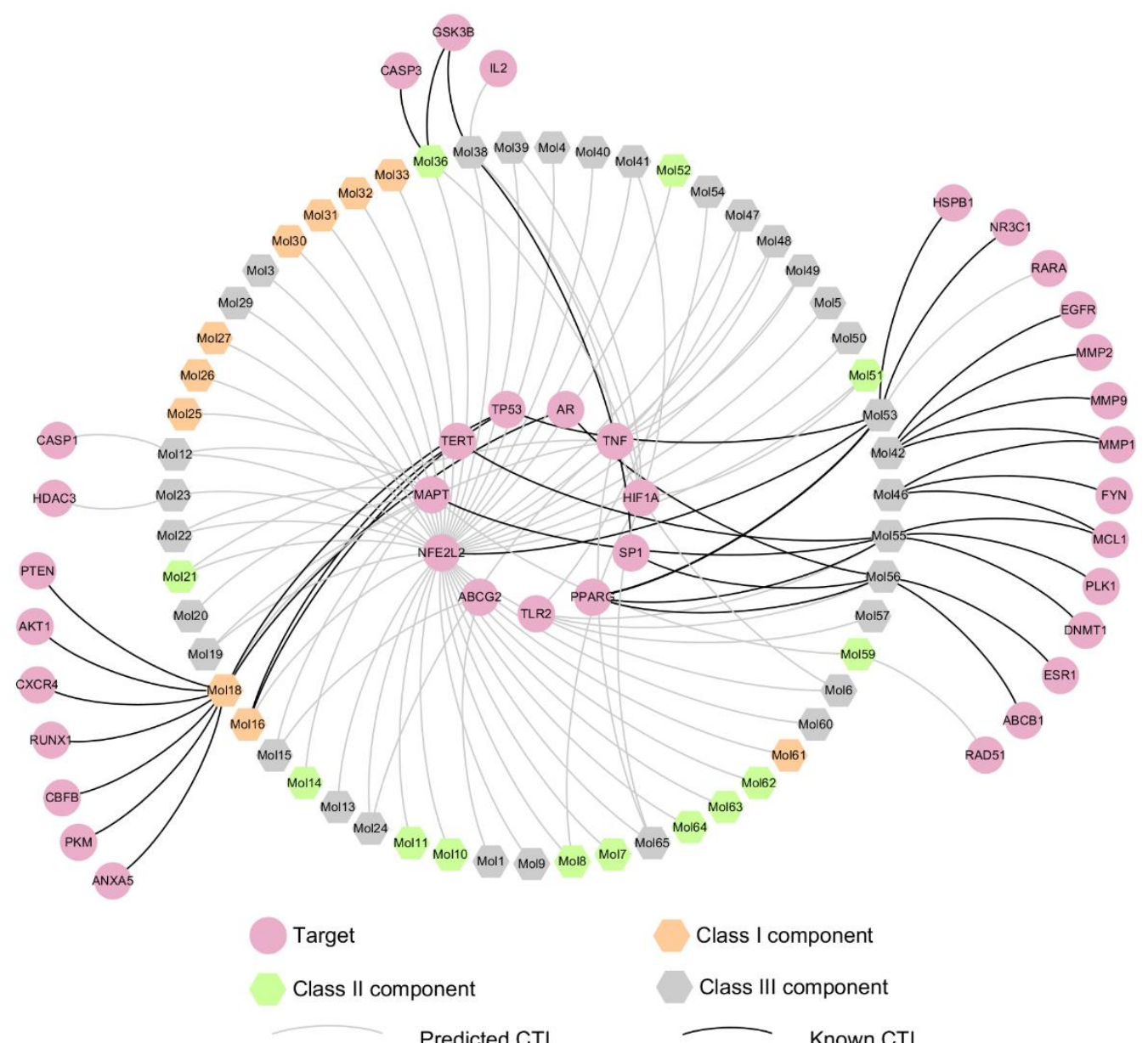

Figure 3. Weighted compound-target bipartite network. Pink circle nodes represent potential targets, hexagon nodes remark components and each edge represent the interaction between them. Orange hexagon nodes represent Class I components, which have the highest concentration; green hexagon nodes remark Class II components, which have a lower concentration; gray hexagon nodes remark Class III components, which have without concentration data. Silver edges represent predicted CTIs and black edges remark known CTIs. 


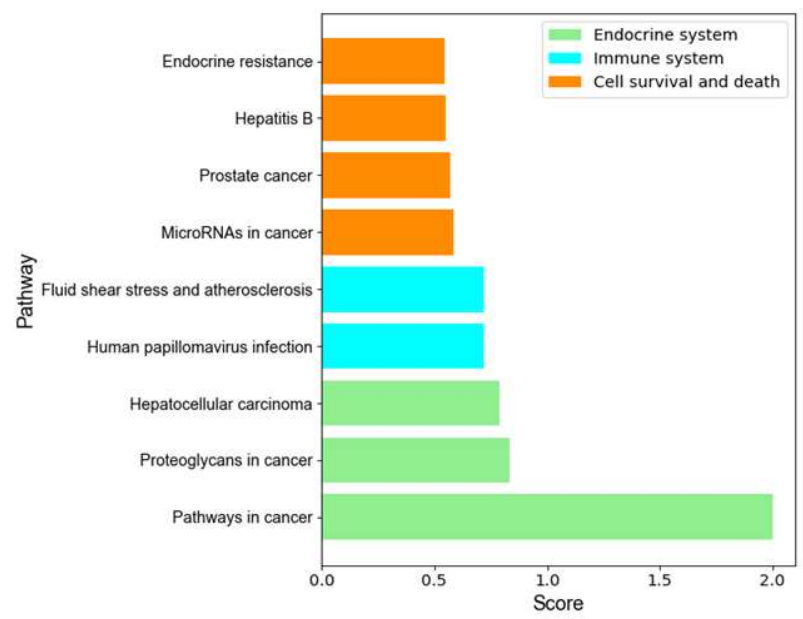

a

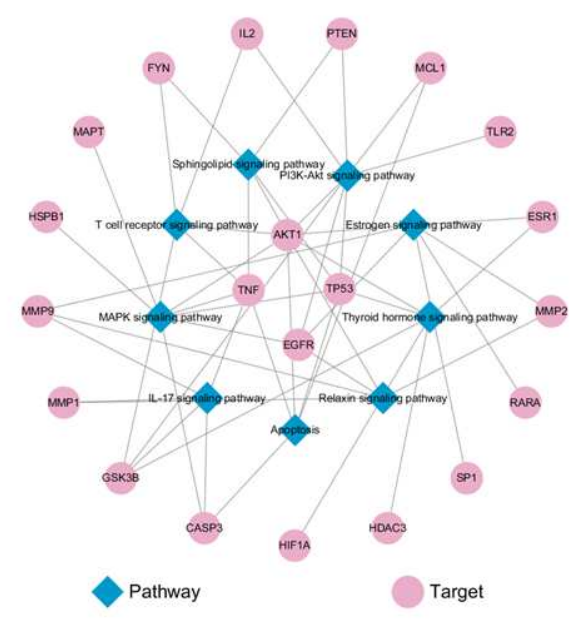

b

Figure 4. KEGG pathway analysis. (a) the score of 9 selected KEGG pathways. 9 pathways were divided into three categories, including four cell survival and death pathways (orange), two immune system pathways (cyan), three endocrine system pathways (green); (b) the selected 9 KEGG pathway-target interaction. 


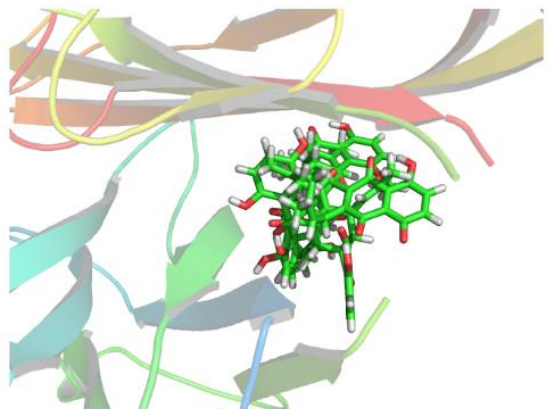

(a)

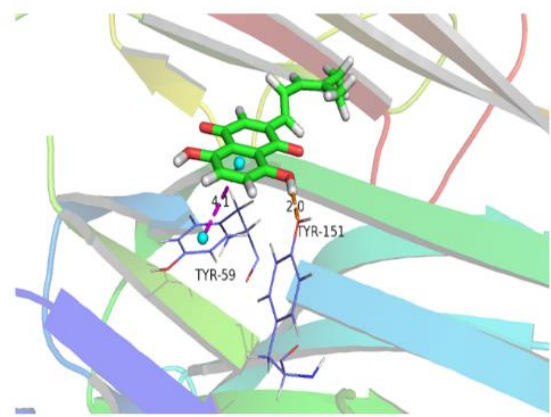

(c)

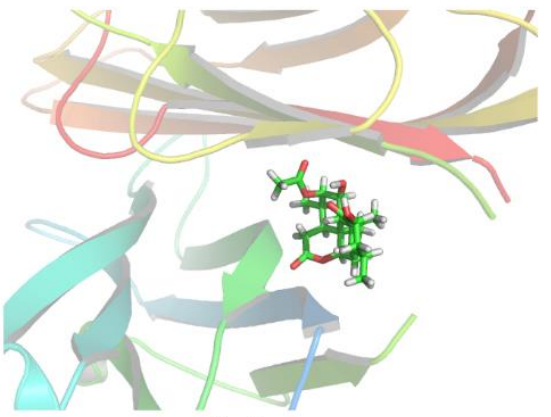

(e)

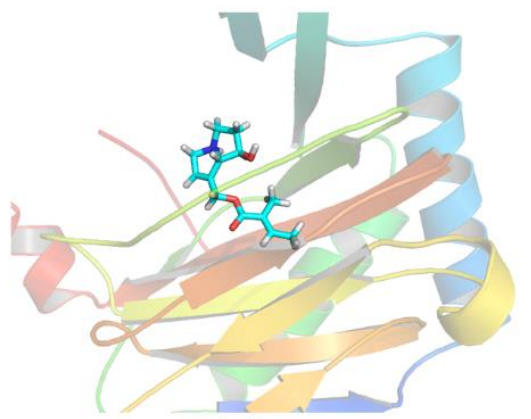

(g)

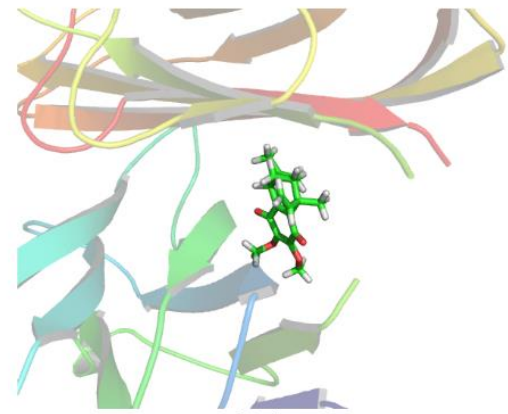

(b)

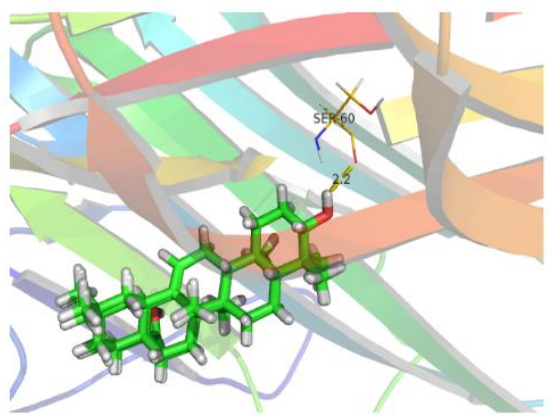

(d)

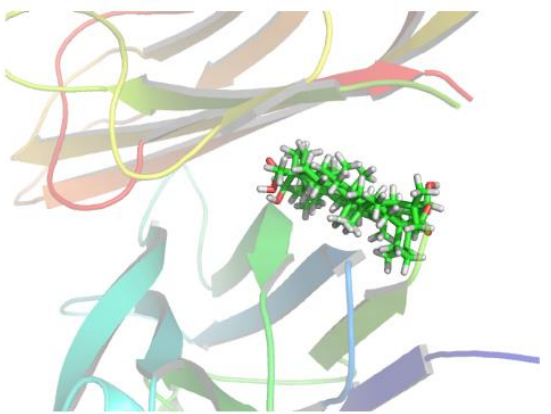

(f)

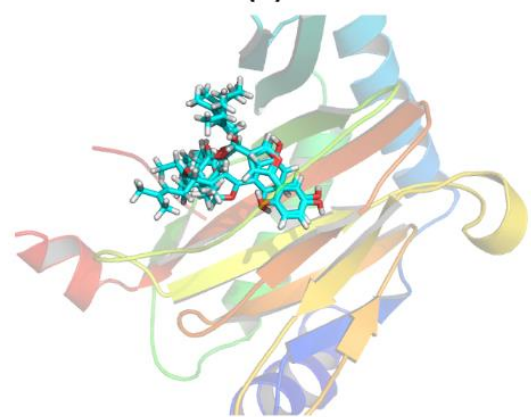

(h)

Figure 5. The interactive modes of 2 targets and the corresponding 8 compounds. (a) arnebinone to TNF (docking score $=-6.222$ ); (b) arnebiabinone to TNF (docking score $=-5.658$ ); (c) dehydroalkannin to TNF (docking score $=-5.405)$; $(\mathrm{d})$ oleanolic acid to TNF (docking score $=-4.875)$; (e) shinjulactone $\mathrm{k}$ to TNF (docking score $=-4.875$ ); (f) ursolic acid to TNF (docking score $=-4.789$ ); (g) shikonofuran $b$ to HIF1A (docking score = -4.802); (h) o9-angeloylretronecine to HIF1A (docking score = -4.488). 


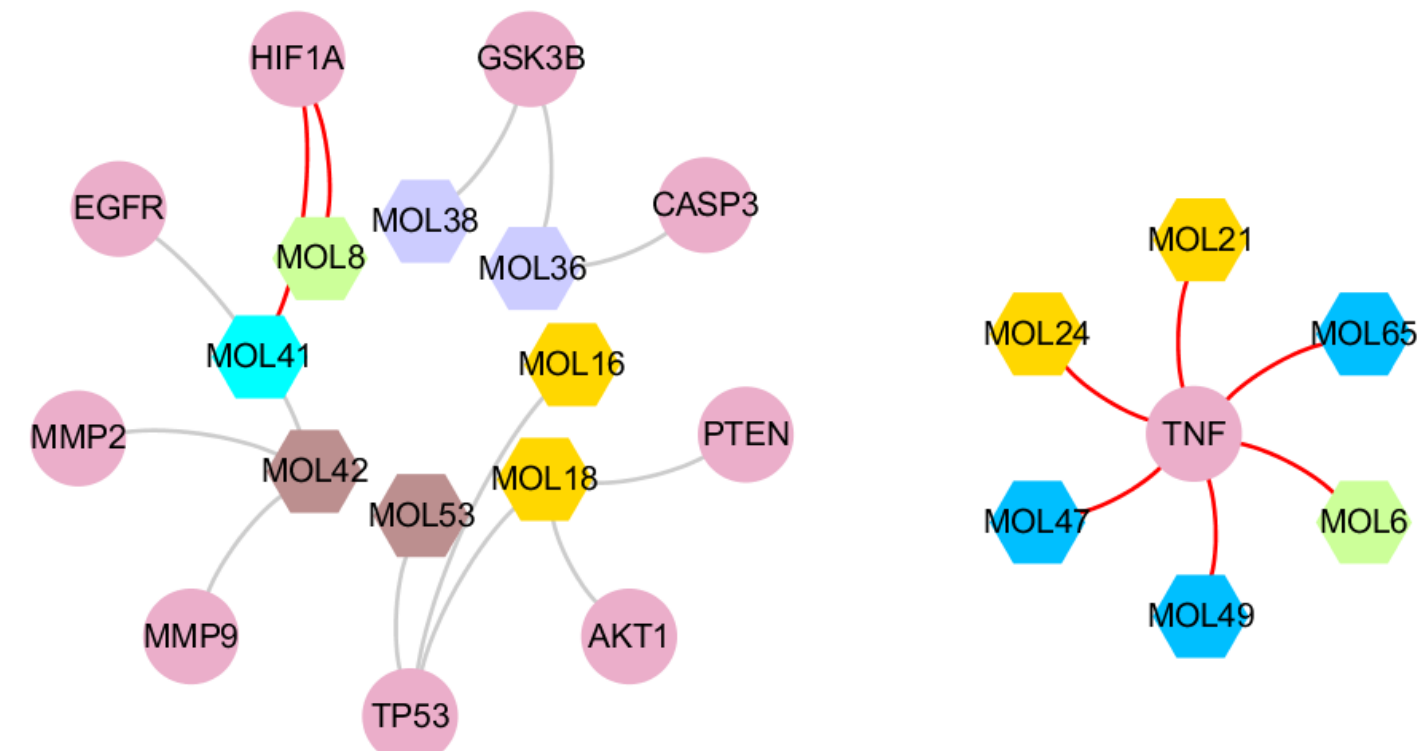

Target

Naphthoquinone Monoterpene phenol and
benzoquinone

Alkaloid

Steroid

Organic acid

Triterpenoid

\section{Known CTI}

Predicted CTI

Figure 6. Critical compound-target bipartite network. Pink circle nodes represent critical targets, hexagon nodes remark components and each edge represent the interaction between them. Orange nodes represent naphthoquinones; green nodes remark monoterpene phenol and benzoquinones; cyan nodes remark alkaloids; purple nodes represent steroids; brown nodes remark organic acids; blue nodes represent triterpenoids. Silver edges represent known CTIs and red edges remark known CTIs. 
Figures
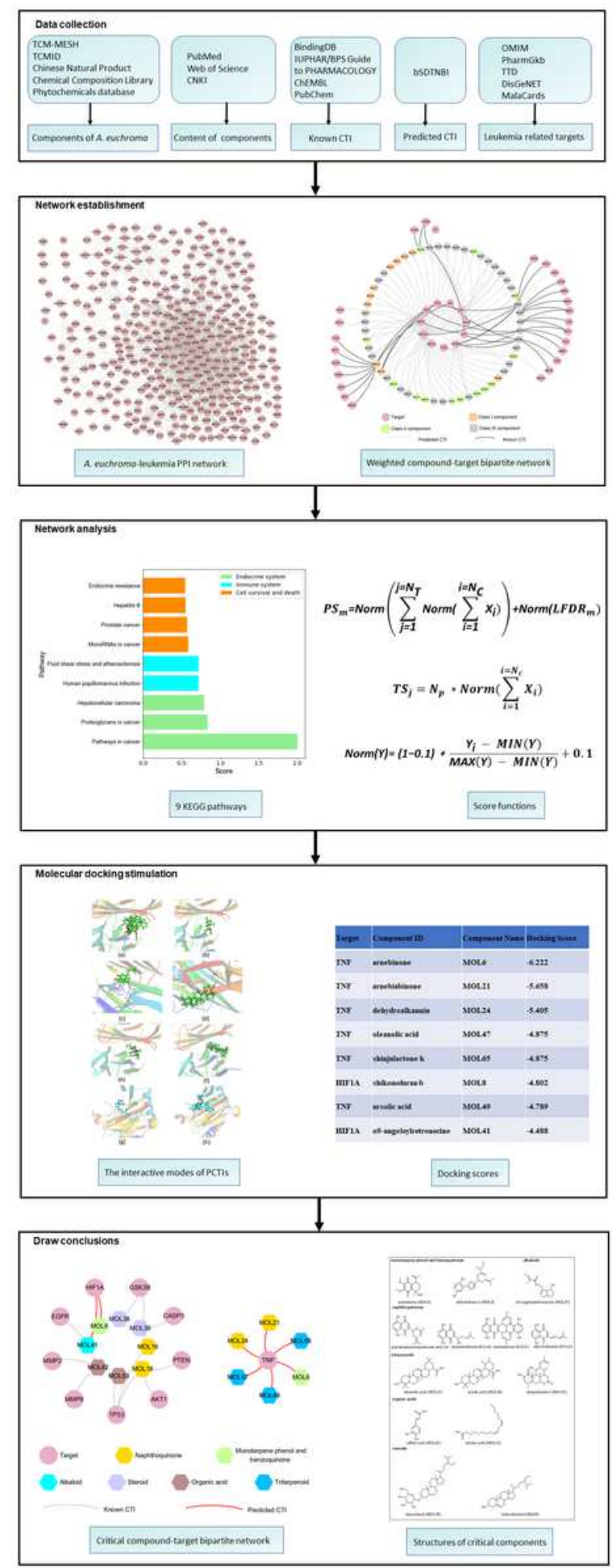

Figure 1

Workflow for A. euchroma on leukemia. 


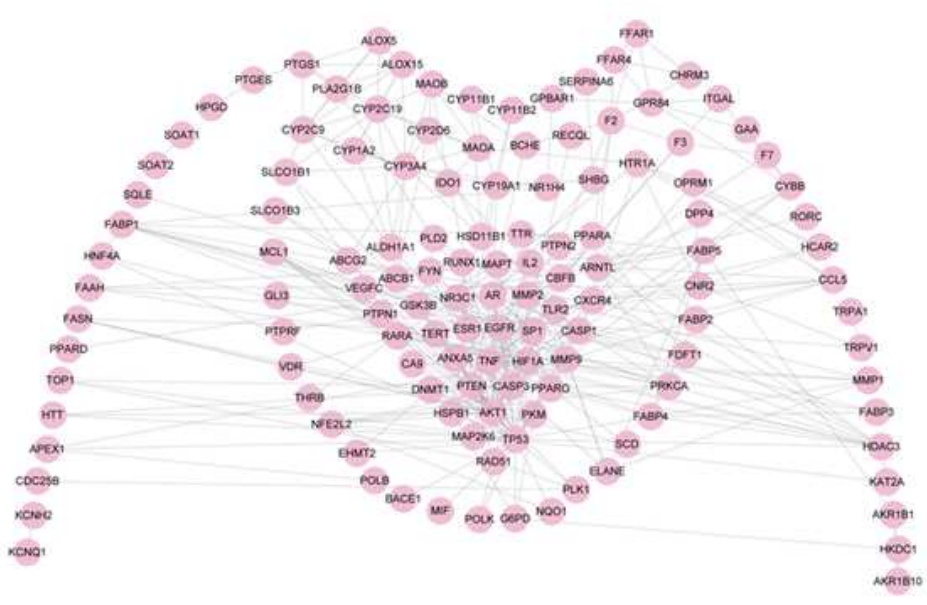

A. euchroma PPI network

(a)

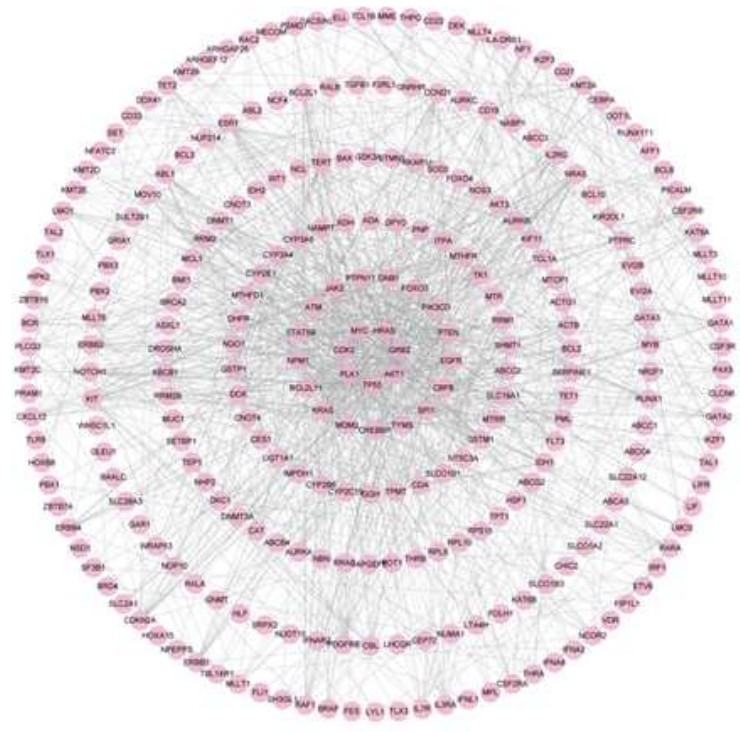

Leukemia PPI network

(b)

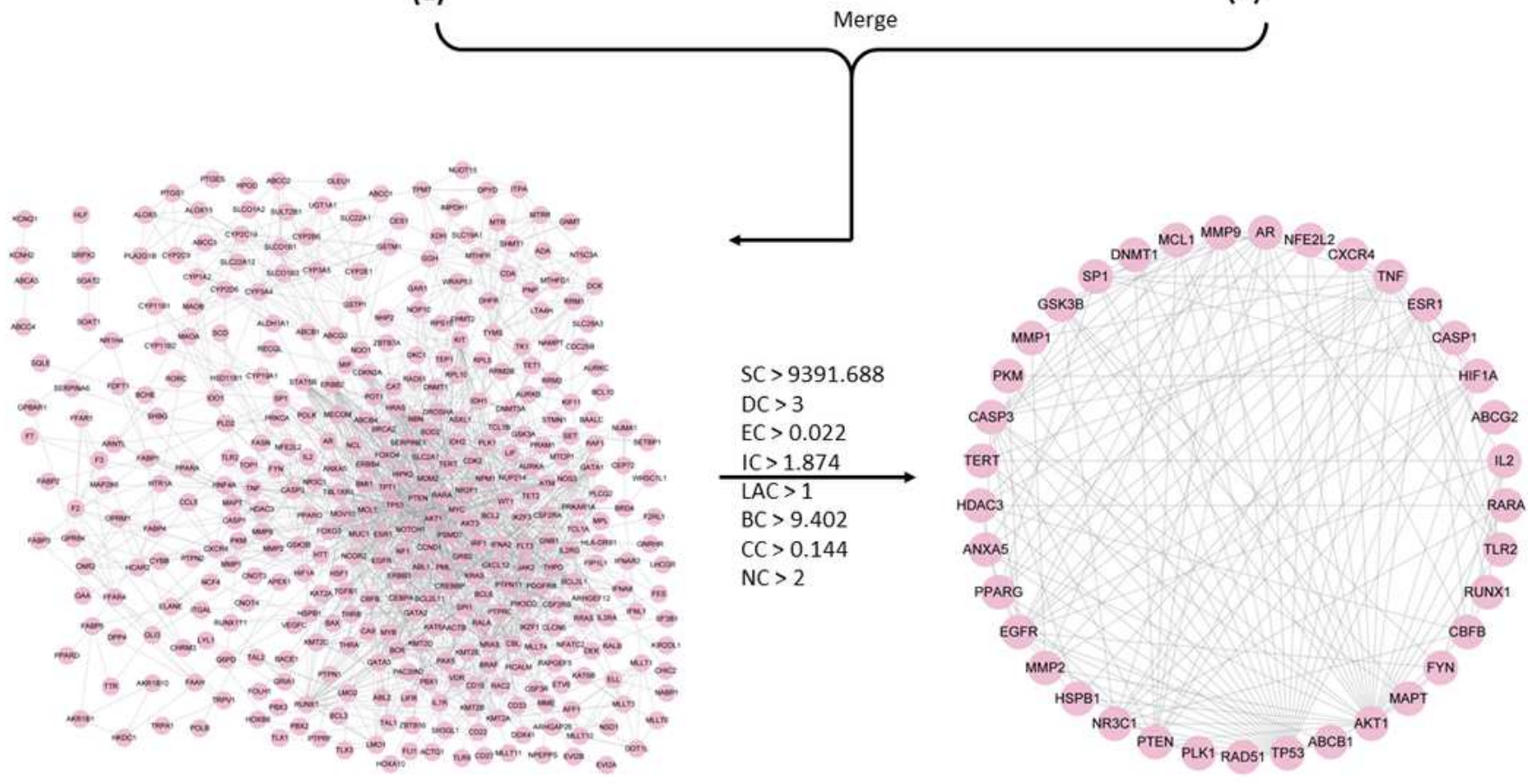

A. euchroma-leukemia PPI network

(c)

Selected targets of $A$. euchroma

(d)

\section{Figure 2}

Construction of A. euchroma-leukemia PPI network. (a) A. euchroma PPI network (PPIA); (B) Leukemia PPI network (PPIL); (C) A. euchroma-leukemia PPI network; (D) Targets of A. euchroma that meet the screening criteria. 


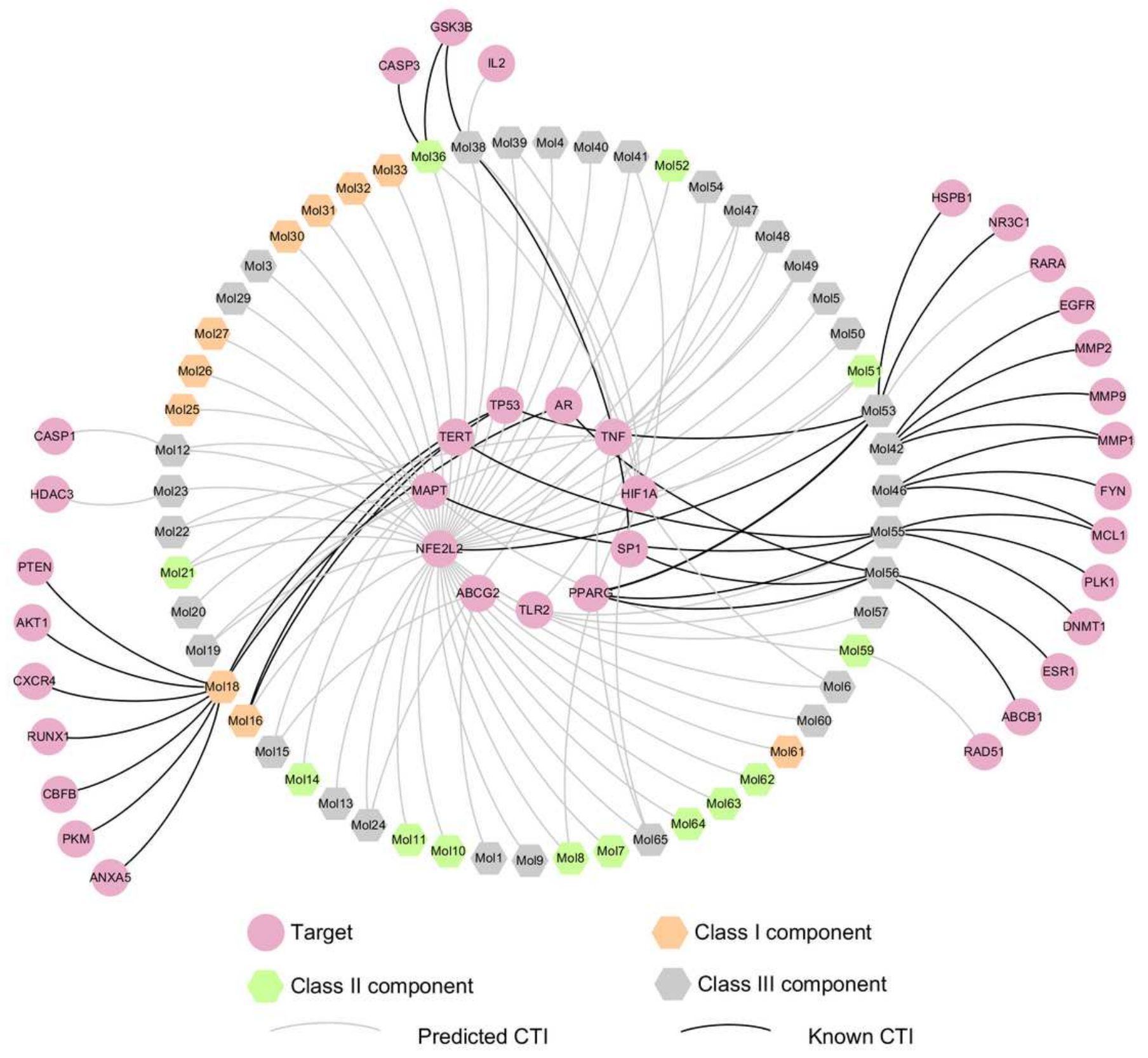

\section{Figure 3}

Weighted compound-target bipartite network. Pink circle nodes represent potential targets, hexagon nodes remark components and each edge represent the interaction between them. Orange hexagon nodes represent Class I components, which have the highest concentration; green hexagon nodes remark Class II components, which have a lower concentration; gray hexagon nodes remark Class III components, which have without concentration data. Silver edges represent predicted CTIs and black edges remark known CTIs. 


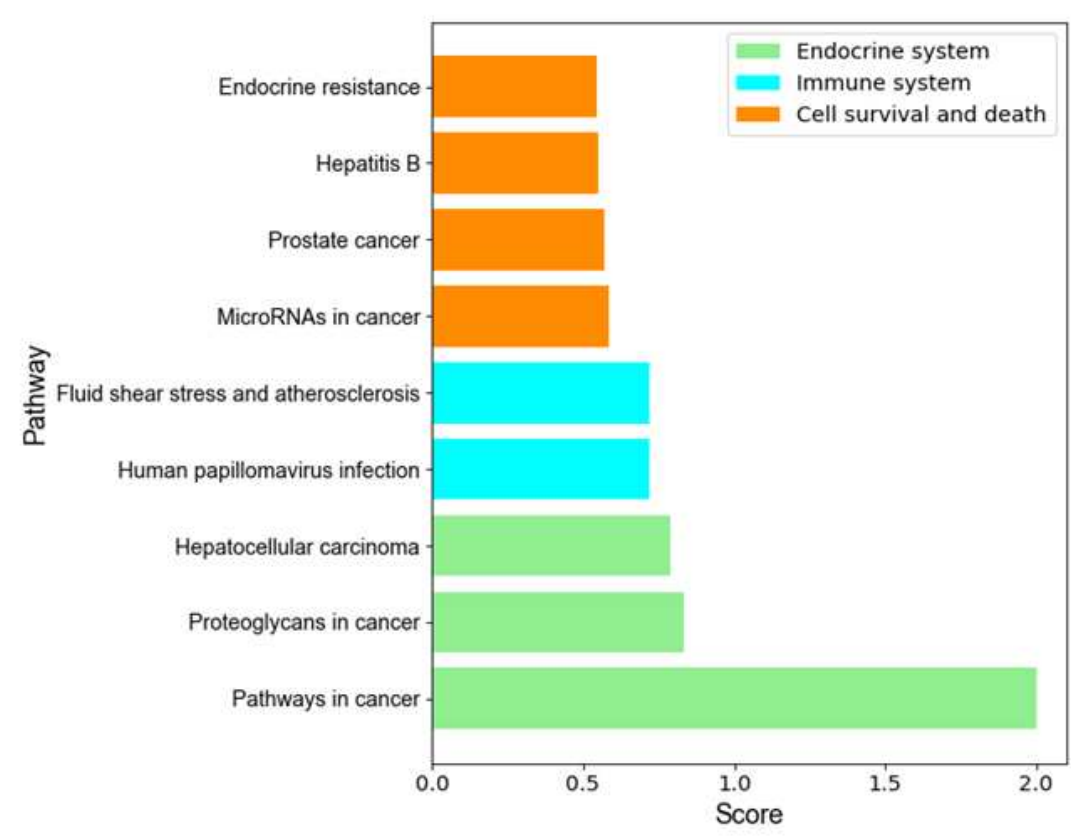

a

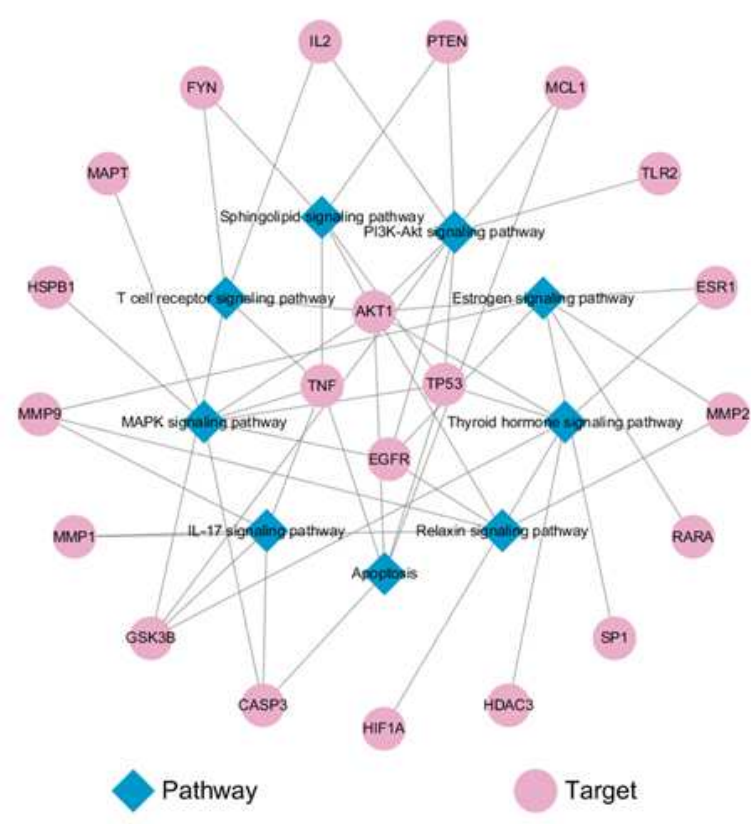

b

Figure 4

KEGG pathway analysis. (a) the score of 9 selected KEGG pathways. 9 pathways were divided into three categories, including four cell survival and death pathways (orange), two immune system pathways (cyan), three endocrine system pathways (green); (b) the selected 9 KEGG pathway-target interaction. 


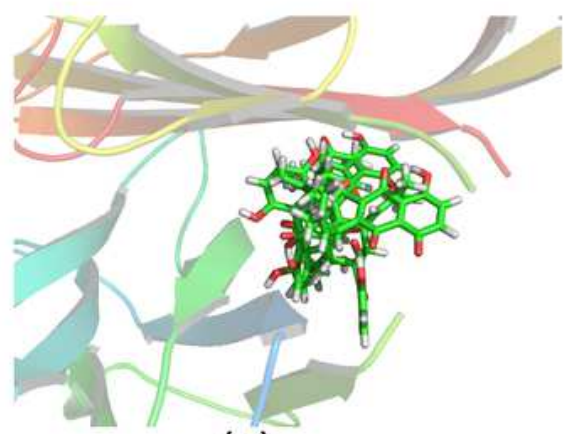

(a)

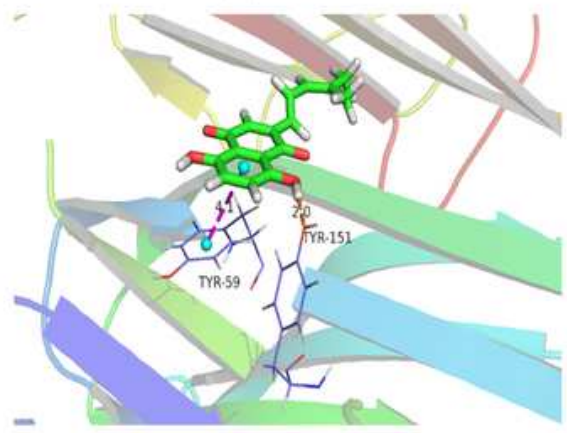

(c)

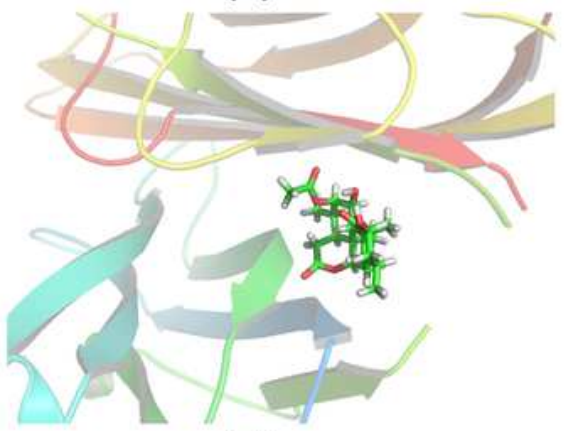

(e)

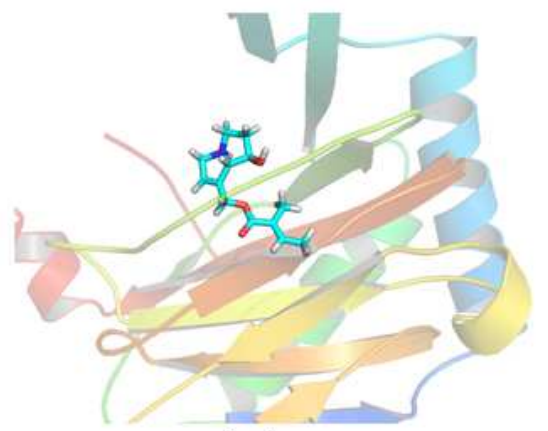

(g)

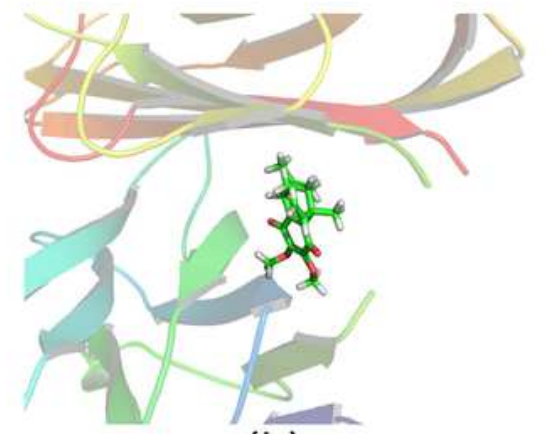

(b)

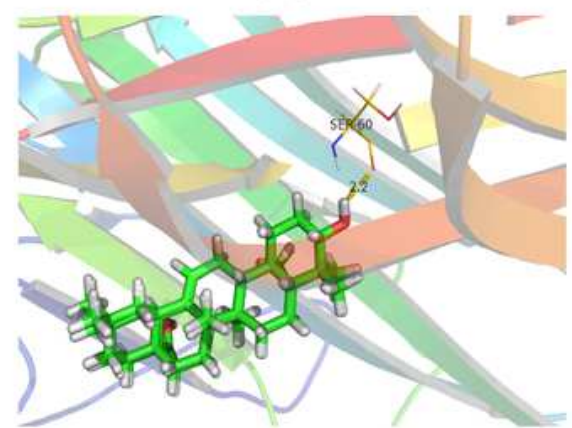

(d)

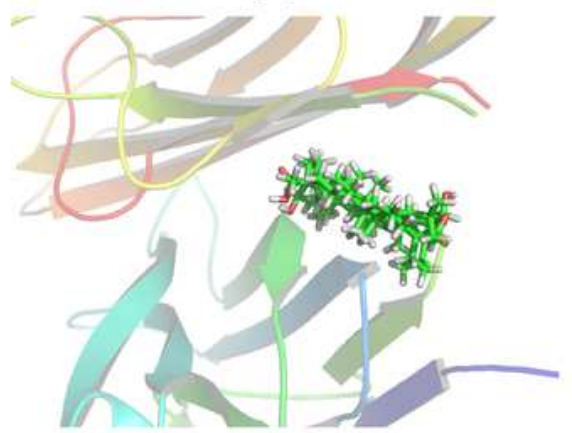

(f)

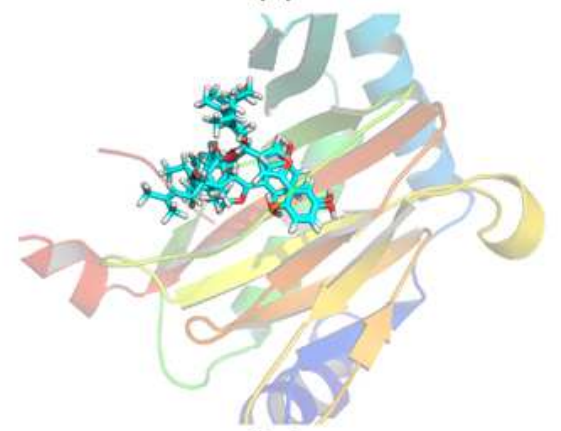

(h)

\section{Figure 5}

The interactive modes of 2 targets and the corresponding 8 compounds. (a) arnebinone to TNF (docking score $=-6.222$ ); (b) arnebiabinone to TNF (docking score $=-5.658$ ); (c) dehydroalkannin to TNF (docking score $=-5.405$ ); (d) oleanolic acid to TNF (docking score $=-4.875)$; (e) shinjulactone $k$ to TNF (docking score $=-4.875$ ); (f) ursolic acid to TNF (docking score $=-4.789$ ); (g) shikonofuran b to HIF1A (docking score $=-4.802$ ); (h) 09-angeloylretronecine to HIF1A (docking score $=-4.488$ ). 


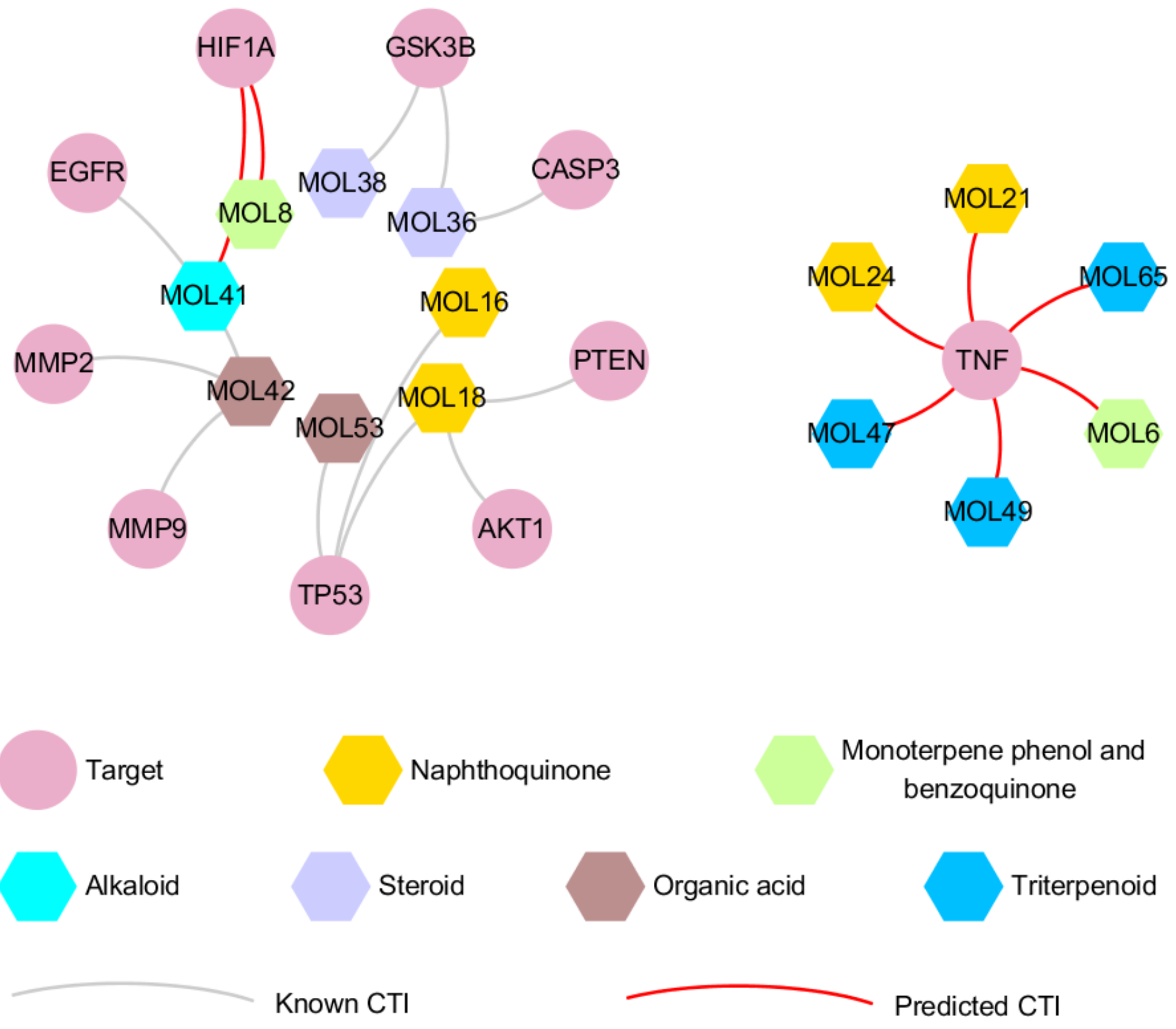

Figure 6

Critical compound-target bipartite network. Pink circle nodes represent critical targets, hexagon nodes remark components and each edge represent the interaction between them. Orange nodes represent naphthoquinones; green nodes remark monoterpene phenol and benzoquinones; cyan nodes remark alkaloids; purple nodes represent steroids; brown nodes remark organic acids; blue nodes represent triterpenoids. Silver edges represent known CTIs and red edges remark known CTIs.

\section{Supplementary Files}

This is a list of supplementary files associated with this preprint. Click to download. 
- FigureS2.png

- SupplementaryTables.xIsx

- FigureS1.png 